\title{
IS THE MUCH DISCUSSED AGILITY OF CORPORATE REAL ESTATE VISIBLE IN PRACTICE? AN EMPIRICAL STUDY OF THE RELATIONSHIP BETWEEN BUSINESS METRICS AND SURPLUS PROPERTY
}

\author{
Howard COOKE ${ }^{1,2, *}$, Rianne APPEL-MEULENBROEK ${ }^{1}$, Theo ARENTZE ${ }^{1}$ \\ ${ }^{1}$ Department of the Built Environment, Eindhoven University of Technology, PO Box 513, 5600 MB Eindhoven, \\ The Netherlands \\ ${ }^{2}$ CORE Consult, 69 Derwent Drive, Maidenhead, Berkshire SL6 6LE, UK
}

Received 19 May 2018; accepted 27 November 2018

\begin{abstract}
Very little work has been undertaken on the consequences of economic recession on Corporate Real Estate (CRE) and its realignment following strategy changes. Only those CRE portfolios with short term leases have a dynamic alignment capability allowing them to readily adjust to change. For those with longer leases this leads to the creation of a surplus property provision (SPP). This paper analyses the relationship between SPP and metrics for business and CRE through a period of significant change, by examining company annual reports using a distributed time lag auto-regression model. The results show an inverse relationship between SPP and profits but a positive relationship with both turnover and employment, suggesting that declining profits trigger the re-shaping of CRE. SPP is used to provide portfolio flexibility because of the lack of dynamic alignment capability. SPP increases as the commitment to short leases $(<5$ years $)$ increases. The estimated time for SPP to revert to zero ranges from 3 to 9 years, but one category, financial services, is continuing to increase its liability. CRE agility has yet to be visible in the financial reports of companies, suggesting its impact remains limited, indicating the relationship between business parameters and CRE is more complicated than envisaged.
\end{abstract}

Keywords: corporate real estate, CRE dynamic alignment, surplus property provisions, agility, restructuring.

\section{Introduction}

Internal and external factors operate to influence and change an organisation and latterly have impacted the rate of change. Change arises directly from strategic choice, such as business strategy, and from transformation in the external environment, such as economic recession. Indirectly it can be through the likes of generational differences, but change is itself an influencer of business strategy, especially the speed of change. The two dominant business strategy theoretical frameworks since the mid 1980's have been sustained competitive advantage (Porter, 1985) and the Resource-Based View (RBV) (Barney, 1991). Neither consider CRE, despite its importance to the business (O'Mara, 1999). Both assume change to be a gradual transition, therefore, CRE re-alignment with a new business strategy would evolve. However, increasingly these strategies have been challenged by more dynamic approaches. Transient competitive advantage (McGrath, 2013) assumes a short-lived competitive advantage in which business must continually change to find the next short-term gain.
Whilst blue ocean strategy (Kim \& Maugborgne, 2015) is more radical, stating that true competitive advantage can only be found in areas of new business or by applying new operating methods to existing business areas. Both of these new strategic models require the organisation to be agile and unencumbered by assets and liabilities from previous strategies, including ideas, people and CRE. When change occurs realignment of all resources needs to happen quickly.

Existing CRE alignment models focus on the dated business strategy models of sustained competitive advantage and $R B V$. For example, Nourse and Roulac (1993) and O'Mara (1999) are based on sustained competitive advantage, whilst Gibler and Lindholm (2012) adopted the $R B V$ model. The newer, more dynamic strategies of transient competitive advantage and blue ocean strategy have not found their way into CRE alignment models. Changes to buildings, the way space is used, the impact of ubiquitous technology, generational differences and the requirement for CRE itself (Joroff \& Becker, 2017) all require CRE to

*Corresponding author. E-mail: hc@core-consult.co.uk 
be agile and dynamic re-alignment a continual process. Alignment researchers "tend to advance their own model in isolation" (Heywood \& Arkesteijn, 2018, p. 17), which has created a disconnect leading to a "disordered CRE alignment theory" (Heywood \& Arkesteijn, 2017, p. 144).

The current alignment models are theories (Heywood \& Arkesteijn, 2018) that seek to provide a "better understanding of the complementary structure among corporate real estate strategies on the theoretical level" (Gibler \& Lindholm, 2012, p. 26). These models focus on how CRE strategies impact on the performance of the business, but are not always validated (Heywood \& Arkesteijn, 2018) nor do they extend to the implementation of CRE realignment (e.g. Gibler \& Lindholm, 2012, Figure 2, p. 36). Heywood and Arkesteijn (2018) identified a number of models that include implementing real estate strategy as a factor. However, none of these models examine the actual implementation of the strategy, rather they identify possible changes to CRE practices or strategic real estate options. Consequently, current alignment models exclude the practicalities of their own implementation, in particular the effects that arise from the creation of surplus space by closing operational units. Improvement of CRE alignment theory and how it can operate in the 'real world' is important, especially in bridging the gap between academic theory and practitioners and providing a cohesive approach to the subject (Heywood \& Arkesteijn, 2017).

The three elements of CRE strategy implementation are the acquisition of new space, its asset management and the disposal of unwanted units (Kämpf-Dern \& Pfnür, 2014). A capability for dynamic alignment should enable a CRE portfolio to rapidly re-align to the new business strategy. That should avoid the creation of a surplus property portfolio arising from unwanted space, although it may be impaired by a shortage of suitable new space. There are a number of complexities in acquiring new premises and in the asset management of CRE (Apgar, 2009), but arguably there are greater challenges with the disposal of unwanted space. Surplus property, in particular leasehold space, continues to be a financial drain on a business until lease expiry or the unit is disposed of. As surplus space does not directly impact business operations it has both a low visibility and a low priority within the business. Consequently, CRE managers might only be allocated limited resources to mitigate the problems of surplus property.

Each business is unique in its CRE requirements, including what it regards as core and peripheral (Gibson \& Lizieri, 1999) and in how it structures ownership - the freehold-leasehold split (Haynes \& Nunnington, 2010). In practical terms the disposal of unwanted freehold property generally offers more potential solutions and fewer restrictions than that of a comparable surplus leasehold. Both have to deal with the effects of market demand together with internal financial constraints and issues, such as writing off the costs of fit-outs. The disposal of surplus leases has the added complexity of the involvement of the landlord and their consent to subletting etc. which has an effect on the cost and risk profiles. Whilst the lease provides the framework for disposal decision-making it does not provide certainty. The reality is that the landlord is the ultimate decision maker, despite the interests of the two parties not being aligned; often even polar opposites. In practice a landlord will most likely primarily focus on asset value with cashflow probably a secondary consideration, whereas for the occupier cashflow is likely to be the primary consideration, followed by any write-off of capital investment. Consequentially, a tenant's proposal to solve a surplus leasehold problem will have to be acceptable to the landlord. Principally this will need to improve the asset value, whilst compensating for any cashflow differential over the current lease term. Even with such a holistic solution there is no obligation on the landlord to act collaboratively or in a logical manner. The landlord has the potential to delay or prevent transactions, whilst actions to force a landlord are generally commercially unviable. There is a lack of research into the behaviour of both landlords and tenants in the operation of lease clauses, despite the impact it has on the operation of leases. The limited literature is focussed on specific issues, such as break clauses (e.g. Cooke \& Woodhead, 2008).

Surplus leaseholds have been an issue for a number of years in the UK reflecting the traditional long lease structure (Cooke, 2004). The limited research on the subject indicates an increasing rather than decreasing problem for FTSE350 companies (the largest 350 companies by market capitalisation listed on the London Stock Exchange) with the surplus property provision (SPP) rising from $£ 1$.6bn in 2007 to $£ 4$ bn in 2014 (Cooke \& Appel-Meulenbroek, 2015). The paucity of research reflects wider issues around CRE research, which includes; a relatively limited number of CRE researchers (Heywood, 2011), the omission of a cross disciplinary approach in CRE (Lizieri, 2003), a lack of corporate strategy researchers considering CRE issues and seemingly no CRE researchers looking at business (Roulac, 2001). The limited and generally dated research on surplus property has focussed on disposal of freeholds (e.g. Gale \& Case, 1989), considered specific categories (e.g. Avis \& Dent, 2004 on the NHS), assessed the scale of the issue (e.g. Cooke \& Appel-Meulenbroek, 2015), considered the practical issues of the problem (e.g. Cooke \& Foster, 2016) or examined accounting regulation change (e.g. Maiona, 2013). The limited research reflects the lack of attention the subject receives academically and professionally, despite it being a key factor in the success of business change and CRE realignment. Intrinsic to that success of CRE realignment is the removal of 'unwanted properties' together with their drain on cash and management resources.

This paper poses hypotheses which are tested with the aim of understanding the relationship between business and CRE parameters and the surplus leasehold portfolio, including whether individual categories of business (e.g. retail, financial services, etc.) behave differently. It examines empirical data drawn from financial reports of companies, including business data (turnover, profitability 
and employment numbers) and CRE data (lease profile and the SPP made for surplus leasehold property) over an eight-year period. The primary analysis is based on a distributed time lag auto-regression model.

The remainder of the paper is structured as follows. The following section discusses the literature with respect to the question how CRE operational requirements can alter as business changes and the creation of a surplus portfolio when CRE requirements change. Next, the research questions are formulated and the adopted methodology described. Then, in the following section the results and discussion are presented. The paper concludes with a discussion of limitations, issues for practitioners and suggestions for further research.

\section{Corporate real estate management and surplus property provision}

Neither of the two business strategy models used by CRE researchers expressly state that CRE is a resource of an organisation. Although resources are regarded as assets they are rarely seen as a source of competitiveness. The most common real estate strategy, especially for larger firms, remains that of cost reduction, which Gibler and Lindholm (2012) identified as the number one priority for $31 \%$ of CRE Managers, with productivity and flexibility someway behind at $10 \%$ each. When assets are bundled or linked within the organisation, they can drive superior performance (MacIntosh \& MacLean, 2015) but they can also create rigidity (Bingham, Eisenhardt, \& Furr, 2011) and impair dynamic capability to react and adjust to a new business strategy. CRE is a resource which can create rigidity and impair the organisation if dynamic alignment capability is not built in. The change from a static to an agile portfolio was identified by Joroff and Becker (2017) as one of the primary shifts in CRE over the last two decades. Indeed, they identified it as a paramount objective. The ability to adjust CRE to provide this agility requires a dynamic alignment capability. The link between business and CRE decision-making is important because "effective real estate decisions are integral to the realisation of overall business objectives" (Nourse \& Roulac, 1993, p. 476).

The development of a business strategy is not a defined process, rather it is an iterative, emergent and adaptive one (e.g. Mintzberg, Ahlstrand, \& Lampel, 2009; Kay, 2010). If this iterative process, with feedback loops, has been adopted both the organisation and all of its managers should have learnt about the risks that CRE rigidity poses and, consequently, have amended their business decision-making process. Business should have built flexibility into its CRE through dynamic alignment capability, enabling it to exploit opportunities and avoid creating surplus property. A holistic view of strategy decision-making is required as moving away from a snapshot approach will remove the risk of overlooking "important seismic shifts in business" (Krumm \& de Vries, 2003, p. 66). It will allow the likes of the longevity of CRE lease decisions to be taken into consideration.
A common method of categorisation of CRE is by cost and increasingly internal performance measures (Riratanaphong \& Van der Voordt, 2015). However, occupiers also need to classify it from defensive and offensive perspectives. Defensively the business needs to know in advance which units it will close if there is a downturn requiring a reduction in CRE. Whilst CRE flexibility allows the smooth integration of new business acquisitions or changing to more suitable premises. The decision-making process needs to be structured with these points in mind and implemented for each CRE decision thereby building dynamic alignment in to the portfolio.

Gibson and Lizieri (1999) proposed a framework for CRE to reflect the changes to new employment structures and working practices. They identified the core and periphery elements of CRE. The core portfolio is characterised by properties that the business needs to control all aspects of, including an ability to change, which will comprise the likes of the HQ and manufacturing plant, and will be owned or held on long leases. Periphery properties make up the remainder of the portfolio and comprise two periphery elements. Those required for one to five years will be held on short leases ( $<5$ years), whilst project space will be on very flexible pay as you go arrangements (e.g. serviced offices). Such an approach provides the CRE Manager with a blueprint for implementing change to the CRE.

An often-cited approach to assessing the worth of $\mathrm{CRE}$ is its added value which is identified as a decisionmaking driver in a number of CRE alignment papers (e.g. Lindholm, Gibler, \& Levainen, 2006). The definition of added value is used loosely and does not appear to be grounded in the economic concept of economic value added (EVA) (Worthington \& West, 2001), a more exact and complex definition. Yet for CRE a significant consideration is understanding all of the ramifications of the effects a change to CRE strategy has, as the impact will not be as simple as an increase in the market value of a property. Jensen, van der Voordt, and Coenen (2012) identified the need for a more holistic solution and a number of models have been reviewed by Heywood and Arkesteijn (2017) who identified various short comings. Van der Voordt, Jensen, Hoendervanger, and Bergsma (2016) created the Value Adding Management model which assumes a continuous cyclical process of Planning, Doing, Checking and Acting. This is illustrated as a closed loop system which provides internal feedback, but does not explicitly show where external factors, such as the business environment and the business itself, can influence the models' parameters. Whilst they may be dealt with by evaluating circumstances a schematic that shows a closed loop system conveys a sense that CRE operates in isolation from the business itself. There has not been the recognition that business is a complex adaptive system (Reeves, Levin, \& Ueda, 2016), and CRE is part of that broader ecosystem. Another limitation of CRE alignment models is that they are office-centric and omit other property types, to the detriment of the overall debate and engagement with practitioners. 
To identify any added value there is a need to measure CRE performance and the effects of change over time. However, CRE performance measurement predominantly comprises input indicators (e.g. operating costs) with insufficient attention paid to those measures that affect strategic decision-making (de Vries, de Jonge, \& van der Voordt, 2008). CRE added value is not viewed as a multidimensional concept. In particular, the impact of 'time' is overlooked and the disconnect between the relevant periods such as business strategy cycle ( $<2$ years), the life span of the leases (circa 10 years) and CRE investment write down periods ( $>20$ years). The theory-based approach of CRE alignment to business strategy generally discounts time as a factor. It does so by the simple expediency of ignoring it and assuming it is a one-off event rather than a continual dynamic process. In the same way relocation strategy is not considered in the context of what happens to the existing property. The added value approach too frequently defaults to the lean management ideology of reducing costs as that is readily understood by both property and non-property managers.

Various techniques have been proposed to assess alignment models, for example, the Balanced Scorecard (BSC) (Kaplan \& Norton, 1996). The financial perspective in the BSC matrix should include SPP arising from a strategy change. Underpinning the BSC technique is long-term strategic learning and feedback from the BSC outputs to improve business performance and the BSC itself. To be effective, performance measurement needs to measure the right things and for the output to be relevant in the context of a decision-making process (Neely \& Bourne, 2000). This necessitates a holistic approach to strategy development, decision-making and implementation, including consideration of the entire life cycle to understand the inter-relationship between business and CRE strategy development, decision-making, lease lengths and SPP. It needs to include how the risks and costs of surplus property are mitigated, including their impact on the performance of the business, together with an assessment of the performance of the CRE team in alleviating the SPP impact. The iterative feedback loops within strategy development and decision-making should review decisions and assess the ultimate consequences to correct the criteria for the future, namely take an emergent strategy approach (Mintzberg et al., 2009). This needs to form the basic framework of CRE alignment decision-making.

There appears to be an underlying assumption in alignment models that the added value approach is onesided. Either value is added, or the effect is neutral, with zero influence. This is despite wider discussions on added value itself and omits the idea that a wrong decision on CRE can remove value, namely there can be a negative effect. For example, mis-specifying a production facility can impact production capacity. Creating surplus space can reduce value. If the property is owned writing off fit out costs and writing down asset value negatively impacts business value. In addition, for surplus leasehold property it will necessitate the making of a provision. An example is when the USA IT company Cisco, in anticipation of business expansion, took a number of leases in the Dotcom boom (1997 to 2001) on office units at Green Park near Reading, UK. The planned expansion did not take place and the company only occupied about $25 \%$ of the leased space. The remaining office building of approximately 550,000 sq.ft. remained vacant for over a decade until leases were surrendered close to expiry following a change in ownership of the office park (CoStar, 2012).

Under International Accounting Standard 37 (IAS37, 2001) surplus leases with more than 12 months to expiry require that an SPP is made. A capital sum is required to cover the cost of the liability resulting from what has become a non-operational onerous lease, through to the first exit date (Cooke, 2004). The provision for surplus property is taken in the year of closure and reviewed annually, reducing profits in the year it is first made and in subsequent years when re-provisioning is undertaken. An annual review is required under IAS37 to ensure that the cash drain arising from payment of rents, etc. is topped up as necessary, ensuring the provision remains adequate to cover the liabilities. When an operational leasehold is closed a number of events take place. Firstly, the rent liability is removed from the operating lease profile, with a reduction in the lease total disclosed. Secondly, any remaining book value will be written down to zero. Finally, a provision is made for the liability of the lease (the SPP), assuming it has more than 12 months to an exit date. The SPP is calculated in several stages, firstly the gross liability is calculated based on costs to exit (including rent, service charge, property taxes, dilapidations etc.). Then an assessment of cost mitigation is undertaken based on disposal assumptions (including rents receivable, marketing and other disposal costs). This element is deducted from the gross liability to create the net liability, the SPP.

The SPP is a specific charge that will reduce the profit for the year it is made (or re-assessed). Thus, perversely at the point when the business is under financial pressure a reduction in profits is required because of the deduction for the provision. There is the potential to see a lag between the decline in revenue and/or profits and the timing of the SPP. This reflects the process of assessing issues and determining a revised strategy with CRE change implemented subsequently. Once business recovery is underway any surplus property should be brought back into use before new space is acquired. There should not be an increase in lease liability without the SPP having been removed.

\section{Research questions}

If a business has learnt from previous experience it will have both a flexible organisation and a CRE portfolio capable of dynamic alignment. CRE will be structured to facilitate the rapid implementation of any change, any significant downturn in business and/or change to business strategy will see the organisation shed its shortest lease commitments first. This will begin with the second pe- 
riphery group (pay-as-you-go space), followed by first periphery (short-term leases initially $<1$ year then $2-5$ years) before longer leases in the core portfolio are considered for closure. The greater the dynamic alignment capability the greater the proportion of leased space in the periphery group. A flexible organisation will be able to re-align its CRE to a new business model without the need to create an SPP. Only if change is extensive will leases with an unexpired term closer to five years be affected and with that a need to create an SPP, but because leases are short it will be a temporary phenomenon.

A change to business strategy frequently results from significant internal or external change, such as a recession or disruptive technological innovation; the period 2007-2014 included both. Businesses came under pressure from reduced revenue and/or profitability and from new entrants introducing new products and services (often technology driven) but unencumbered by existing CRE. Data on business metrics and CRE was extracted from the annual financial reports of companies in the FSE350 for that period to test hypothesised relationships in four research questions.

The first research question (Q1) considers whether business and CRE metric profiles differ between those companies that make an SPP and those that do not. We hypothesise that companies that are agile are less likely to create an SPP because they will be able to adjust their CRE to the changing environment (Q1). Larger companies are likely to be less agile and therefore slower to change direction and hence more likely to make an SPP.

The second research question (Q2) considers what triggers the making of an SPP. Our hypothesis is that a decline in turnover and/or profits and/or profit margin and/or fulltime employee numbers (FTE's) indicates a deterioration in the business. This will trigger a cost reduction exercise commensurate with the severity of the decline, which will result in a contraction in production and/or FTE's and/or fixed overheads (including CRE). Consequently, the relationship between SPP and the metrics of turnover, profit, profit margin and FTE numbers are expected to be inverse; SPP will increase as they decline (Q2).

The third research question $(\mathrm{Q} 3)$ considers the relationship between the longevity of the SPP and CRE dynamic alignment. SPP will decline as leases expire, breaks are exercised or surrendered, although in practice, landlords generally only accept a surrender close to a break or expiry date. A decline in SPP might indicate business recovery and re-occupation of surplus space, but in practice businesses have exhibited a reluctance to do so. Operational teams regard such space as 'tainted. The SPP total increases when new surplus properties are added and when re-provisioning for existing surplus properties is required. Initial cuts to CRE should be to pay as you go space followed by short-term leases. If CRE has an inherent dynamic alignment capability it will be structured around a core and periphery model (Gibson \& Lizieri, 1999), therefore the SPP will only exist for a short time (2-3 years). Consequently, the speed that SPP reverts to zero is a measure of CRE dynamic alignment capability (Q3).

The fourth research question $(\mathrm{Q} 4)$ considers the interrelationship between SPP and the lease profile. If business performance declines, there will be a reduction in shorterterm leases $(<5$ years) as an SPP is made. This indicates a structured portfolio with dynamic alignment, whereas a decline in longer leases ( $>5$ years) indicates a lack of CRE dynamic alignment. We hypothesise that a negative relationship between SPP and short-term leases but no relationship between SPP and long-term leases indicates the existence of dynamic alignment of CRE (Q4).

\section{Methodology}

This research examines the annual reports for UK companies quoted in the FTSE350 Index for the period 2007 to 2014 inclusive: the period of the financial crisis and recession. After an initial analysis certain companies were excluded because they were atypical in their use of CRE, including property companies (who own to lease), investment funds (generally virtual organisations from a CRE perspective), natural resource companies (who own land for extraction purposes), health, transport and utility companies (CRE is generally owned) and those who did not have accounts for the entire period. The first stage of analysis splits the dataset between companies that made an SPP in the period and those that did not. As a consequence, the sample size for both the construction and leisure categories became too small ( $<10$ companies) and were excluded from the analysis. This provided a final dataset of 170 companies split in 99 SPP companies (those companies that make a surplus property provision) and 71 non-SPP companies (those that do not make a provision at all in the eight year period).

The analysis consolidated the companies into broad business categories, each with a mixture of property types:

- Finance - include offices and retail, the bank branch network;

- Professional Services - include offices and storage facilities;

- Technology, Media and Telecommunications (TMT) - comprises offices plus more technical space such as telephone exchanges and data centres;

- Manufacturing - whilst predominantly industrial units will include offices;

- Retail - will primarily be shops but includes offices and the warehouse distribution network.

For all 170 companies a profile was created for business and lease metrics by rebasing the data to 100 in 2007 to illustrate the general trends for the metrics over the eight year period. The second stage was to provide a broad context for the different categories split between SPP companies (those that make a surplus property provision) and non-SPP (those that do not make a provision). This was carried out by using an independent samples $t$-test of differences in means in 2014 of the business and CRE metrics between the SPP and non-SPP groups. A further $t$-test was 
carried out on the percentage change between 2007 and 2014 for variables. This analysis allowed us to examine the business and CRE metrics over the period and consider differences between the SPP and non-SPP groups (Q1). The sample sizes for the non-SPP categories of Retail, TMT and Professional Services are relatively small, which raises the question as to the reliability of the outcomes of the $t$-test. However, this does not necessarily invalidate the test because a sample of 8 companies is not regarded as very small $(\mathrm{N}<5)$ (de Winter, 2013). Although the test works for small samples there is a question as to the extent to which a small sample like this is representative for the companies in the sector. This is a consideration to be kept in mind. The only very small category was Retail non-SPP (4 companies) and the $t$-test does not indicate any significant relationship between the two groups for this category.

The non-SPP can be categorised into three sub-sections: (i) companies that do not have surplus leases; (ii) those with surplus leases but deemed it insignificant to require an SPP; and (iii) those with surplus leases in their total provision, but do not identify SPP separately from their overall provision. As there is not the information available to determine the exact status an assumption has been made that if a company does not state an SPP figure it is because they do not have any surplus leasehold property. The 71 non-SPP companies have been excluded from the subsequent regression analysis as the SPP is zero for that group for all years.

The primary analysis technique used on the SPP companies was a distributed time lagged auto-regression model. The model includes SPP as the dependent variable and the previous year's SPP value as the auto-regression term to take the time-series nature of the data into account. The independent variables are turnover, profit, profit margin, FTE's, net assets, owned CRE and operating leases (grouped into leases $<1$ year, leases $2-5$ years and leases $>5$ years). The profit figure adopted was the stated profit less any increase in the SPP for that year. Any increase in SPP will decrease the profit for that year, therefore, to identify the underlying profits for the business the effect of that deduction needs to be removed by adding back in the increase in the SPP. Profit margin is calculated using the adjusted profit expressed as a percentage of turnover. In addition, the Total CRE was calculated (lease total plus the owned CRE) allowing us to assess the proportion of the Total CRE that leases comprise.

Regression models were estimated for each category and for the whole portfolio, All Companies, with 2007 as the base year. A high degree of multicollinearity was expected between the metrics which could give rise to estimation problems. To identify the most significant variable amongst those variables that are strongly correlated (a correlation $\geq 0.700$ ), we undertook regression analysis with the metrics individually whilst retaining the auto-regression term and the dummy variables. The variable that provided the highest goodness of fit ( $\mathrm{R}^{2}$ Adjusted) was identified as the most significant variable and selected for the final model. When the regression was run, a further check for the effects of multi- collinearity was undertaken by examining both the Variance Inflation Factor and tolerance level measures. Dummy variables for encoding the year are included as independent variables, one per year, to capture any fixed effects that are year specific as the regression constant and auto-regressions pick up general trends.

For Q2 and Q3 the independent variables were turnover, profit, profit margin and FTE's for each category. The estimated coefficients of the independent variables offer information about the triggers of SPP (Q2). The estimated coefficient of the previous year's SPP (the auto-regression term) offers information on the speed of decline/increase in the SPP (Q3). If the coefficient equals 1.0 the SPP is static, below 1.0 indicates a decline, with $<0.5$ a rapid decline, whereas $>1.0$ indicates that the SPP is increasing. Finally, regressions were run for Q4 with the independent variables consisting of the value of short-term leases $(<1$ year), medium-term leases ( $2-5$ years) and long-term leases ( $>5$ years).

Implementing change to CRE is generally slow but changing a property from operational to non-operational can be virtually instantaneous. An annual report of a company reflects the position at a moment in time, the end of the financial year. However, the actual change to business strategy and CRE can occur at any time during the twelvemonths of the financial year. To factor in an allowance for implementation a one-year lag has been assumed to be a possibility for each independent variable.

Checks were undertaken to verify that the residuals for each regression were normally distributed by examining the histogram of the residuals. For brevity PY has been used for Previous Year throughout and we have identified significance in tables by ${ }^{*} \mathrm{p}<0.1,{ }^{* *} \mathrm{p}<0.05$ and ${ }^{* * *} \mathrm{p}<0.01$

\section{Results and discussion}

\subsection{Descriptive results - question 1}

For the total sample the profile over the eight years is illustrated in Figure 1. It clearly shows the effect of the recession on profits between 2007 and 2010, and whilst

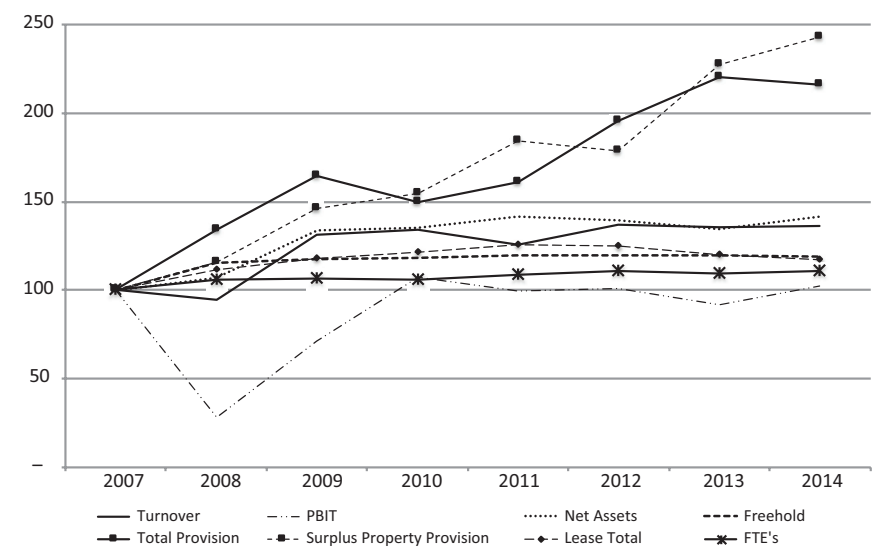

Figure 1. Key measures for all companies $(n=170)$ 
turnover also dropped in 2008 it recovered the following year. The net assets, FTE's and owned and leased CRE remained relatively steady through the period. However, substantial increases were seen with the surplus property provision (SPP) and the total provision, not only in 2008 but continuing through the period.

The analysis of the difference in means between SPP and non-SPP groups are shown in Table 1 and of the percentage changes in Table 2. A number of metrics show a significant difference between the SPP and non-SPP groups, but fewer than anticipated. That appears to be the result of large standard deviations for many of the means. A number of metrics have relatively low $\mathrm{p}$-values, but do not reach the (low) significance level of $\mathrm{p}<0.1$. We will now discuss the results of the analysis. Firstly, we consider the significant differences between SPP and non-SPP means in 2014 followed by the change from 2007 to 2014.

Table 1 . Mean values per company per category in $2014(n=170)$

\begin{tabular}{|c|c|c|c|c|c|c|}
\hline \multirow{2}{*}{ Measure } & \multicolumn{2}{|c|}{2014 mean } & \multicolumn{2}{|c|}{ T-Test non SPP to SPP 2014} & \multicolumn{2}{|c|}{2007 to 2014 change } \\
\hline & SPP & Non-SPP & $t$-value & $\mathrm{p}$-value & SPP & Non-SPP \\
\hline All companies & 99 companies & 71 companies & & & & \\
\hline Turnover (£’m) & 5949 & 4568 & -0.833 & 0.406 & $31 \%$ & $63 \%$ \\
\hline PBIT (£’m) & 483 & 512 & 0.141 & 0.888 & $-16 \%$ & $49 \%$ \\
\hline Profit margin (\%) & $13 \%$ & $16 \%$ & 1.160 & 0.248 & $-4 \%$ & $-7 \%$ \\
\hline Net assets (£’m) & 5246 & 2154 & -1.631 & 0.106 & $40 \%$ & $54 \%$ \\
\hline FTE's & 33124 & 26632 & -0.553 & 0.581 & $11 \%$ & $16 \%$ \\
\hline $\operatorname{SPP}\left(\mathfrak{E}^{\prime} \mathrm{m}\right)$ & 37 & 0 & N/A & N/A & $151 \%$ & N/A \\
\hline Freehold (£’m) & 577 & 233 & -1.399 & 0.165 & $29 \%$ & $24 \%$ \\
\hline Lease total (£’m) & 868 & 267 & -2.598 & $0.010^{\star *}$ & $13 \%$ & $44 \%$ \\
\hline Total CRE (£'m) & 1445 & 500 & -2.088 & $0.039^{* *}$ & $19 \%$ & $34 \%$ \\
\hline Lease \% total CRE (\%) & $71 \%$ & $56 \%$ & -3.224 & $0.002^{\star \star \star}$ & $-3 \%$ & $3 \%$ \\
\hline Lease total as \% years profit (\%) & $51 \%$ & $1208 \%$ & 1.194 & 0.234 & $-90 \%$ & $1850 \%$ \\
\hline Owned CRE per FTE $(\mathfrak{E})$ & 10310 & 12310 & 0.987 & 0.325 & $25 \%$ & $35 \%$ \\
\hline Lease total per FTE $(\mathfrak{E})$ & 44539 & 27382 & -1.279 & 0.203 & $2 \%$ & $78 \%$ \\
\hline Total CRE per FTE $(\mathfrak{E})$ & 54849 & 39692 & -1.106 & 0.270 & $5 \%$ & $62 \%$ \\
\hline Finance & 24 companies & 15 companies & & & & \\
\hline Turnover (£’m) & 9192 & 9106 & -0.016 & 0.987 & $23 \%$ & $91 \%$ \\
\hline PBIT (£’m) & 1130 & 664 & -0.665 & 0.510 & $-28 \%$ & $35 \%$ \\
\hline Profit margin (\%) & $22 \%$ & $20 \%$ & -0.378 & 0.708 & $-13 \%$ & $-27 \%$ \\
\hline Net assets (£’m) & 14671 & 4581 & -1.466 & 0.154 & $53 \%$ & $60 \%$ \\
\hline FTE's & 30915 & 11365 & -1.407 & 0.169 & $-11 \%$ & $1 \%$ \\
\hline SPP (£'m) & 44 & 0 & N/A & N/A & $218 \%$ & $\mathrm{~N} / \mathrm{A}$ \\
\hline Freehold (£'m) & 373 & 114 & -1.510 & 0.141 & $-4 \%$ & $-35 \%$ \\
\hline Lease total (£'m) & 552 & 241 & -1.106 & 0.276 & $-10 \%$ & $29 \%$ \\
\hline Total CRE (£’m) & 925 & 355 & -1.463 & 0.154 & $-8 \%$ & $-2 \%$ \\
\hline Lease $\%$ total CRE (\%) & $81 \%$ & $76 \%$ & -0.637 & 0.528 & $2 \%$ & $-4 \%$ \\
\hline Lease total as \% years profit (\%) & $116 \%$ & $31 \%$ & -2.323 & $0.029^{* *}$ & $130 \%$ & $21 \%$ \\
\hline Owned CRE per FTE $(\mathfrak{E})$ & 6470 & 7880 & 0.528 & 0.600 & $1 \%$ & $-6 \%$ \\
\hline Lease total per FTE $(\mathfrak{E})$ & 40874 & 41387 & 0.036 & 0.971 & $-7 \%$ & $96 \%$ \\
\hline Total CRE per FTE (£) & 47343 & 49267 & 0.128 & 0.899 & $-6 \%$ & $67 \%$ \\
\hline Professional services & 20 companies & 9 companies & & & & \\
\hline Turnover (£’m) & 3015 & 2137 & -0.662 & 0.513 & $54 \%$ & $59 \%$ \\
\hline PBIT (£’m) & 150 & 217 & 0.429 & 0.671 & $6 \%$ & $77 \%$ \\
\hline Profit margin (\%) & $8 \%$ & $14 \%$ & 1.300 & 0.205 & $-15 \%$ & $18 \%$ \\
\hline Net assets (£’m) & 550 & 732 & 0.838 & 0.410 & $28 \%$ & $90 \%$ \\
\hline FTE's & 45888 & 90819 & 0.768 & 0.449 & $32 \%$ & $31 \%$ \\
\hline SPP (E’m) & 14 & 0 & N/A & N/A & $41 \%$ & N/A \\
\hline Freehold (£’m) & 60 & 40 & -0.704 & 0.488 & $13 \%$ & $30 \%$ \\
\hline Lease total (£’m) & 295 & 131 & -0.76 & 0.454 & $48 \%$ & $36 \%$ \\
\hline Total CRE (£’m) & 355 & 172 & -0.863 & 0.396 & $41 \%$ & $35 \%$ \\
\hline
\end{tabular}


End of Table 1

\begin{tabular}{|c|c|c|c|c|c|c|}
\hline \multirow{2}{*}{ Measure } & \multicolumn{2}{|c|}{2014 mean } & \multicolumn{2}{|c|}{ T-Test non SPP to SPP 2014} & \multicolumn{2}{|c|}{2007 to 2014 change } \\
\hline & SPP & Non-SPP & $t$-value & $\mathrm{p}$-value & SPP & Non-SPP \\
\hline Lease \% total CRE (\%) & $74 \%$ & $74 \%$ & 0.014 & 0.989 & $0 \%$ & $12 \%$ \\
\hline Lease total as \% years profit (\%) & $244 \%$ & $72 \%$ & -0.831 & 0.413 & $18 \%$ & $17 \%$ \\
\hline Owned CRE per FTE (£) & 5934 & 3042 & -0.786 & 0.439 & $43 \%$ & $-13 \%$ \\
\hline Lease total per FTE (E) & 29676 & 6724 & -0.909 & 0.371 & $18 \%$ & $9 \%$ \\
\hline Total CRE per FTE (£) & 35610 & 9766 & -1.019 & 0.317 & $22 \%$ & $1 \%$ \\
\hline TMT & 17 companies & 8 companies & & & & \\
\hline Turnover (£’m) & 5757 & 833 & -2.083 & $0.053^{*}$ & $21 \%$ & $142 \%$ \\
\hline PBIT (£’m) & 305 & 152 & -0.313 & 0.757 & $7 \%$ & $140 \%$ \\
\hline Profit margin (\%) & $17 \%$ & $18 \%$ & 0.212 & 0.834 & $26 \%$ & $24 \%$ \\
\hline Net assets (£’m) & 5549 & 594 & -0.805 & 0.429 & $6 \%$ & $103 \%$ \\
\hline FTE's & 25799 & 3665 & -2.411 & $0.028^{\star *}$ & $13 \%$ & $30 \%$ \\
\hline SPP (£’m) & 73 & 0 & N/A & N/A & $167 \%$ & N/A \\
\hline Freehold (£’m) & 130 & 27 & -1.282 & 0.213 & $4 \%$ & $52 \%$ \\
\hline Lease total (£’m) & 965 & 100 & -1.743 & 0.100 & $-2 \%$ & $146 \%$ \\
\hline Total CRE (£'m) & 1094 & 127 & -1.807 & $0.089^{*}$ & $-1 \%$ & $117 \%$ \\
\hline Lease \% total CRE (\%) & $80 \%$ & $74 \%$ & -0.553 & 0.585 & $-5 \%$ & $2 \%$ \\
\hline Lease total as \% years profit (\%) & $-216 \%$ & $10124 \%$ & 1.043 & 0.332 & $-115 \%$ & $39006 \%$ \\
\hline Owned CRE per FTE $(\mathfrak{E})$ & 6148 & 9366 & 0.869 & 0.394 & $58 \%$ & $121 \%$ \\
\hline Lease total per FTE $(\mathfrak{E})$ & 67111 & 92796 & 0.309 & 0.760 & $17 \%$ & $135 \%$ \\
\hline Total CRE per FTE (£) & 73259 & 102162 & 0.343 & 0.735 & $20 \%$ & $134 \%$ \\
\hline Manufacturing & 19 companies & 35 companies & & & & \\
\hline Turnover (£’m) & 2723 & 4276 & 1.059 & 0.295 & $55 \%$ & $45 \%$ \\
\hline PBIT (£’m) & 290 & 636 & 1.334 & 0.188 & $31 \%$ & $57 \%$ \\
\hline Profit margin (\%) & $14 \%$ & $14 \%$ & 1.050 & 0.299 & $19 \%$ & $-3 \%$ \\
\hline Net assets (£'m) & 1105 & 1982 & 0.963 & 0.340 & $61 \%$ & $43 \%$ \\
\hline FTE's & 12433 & 22207 & 1.364 & 0.179 & $11 \%$ & $7 \%$ \\
\hline SPP (£’m) & 9 & 0 & N/A & $\mathrm{N} / \mathrm{A}$ & $121 \%$ & N/A \\
\hline Freehold (£'m) & 209 & 332 & 1.076 & 0.287 & $34 \%$ & $48 \%$ \\
\hline Lease total (£’m) & 107 & 232 & 1.113 & 0.272 & $22 \%$ & $47 \%$ \\
\hline Total CRE (£'m) & 317 & 564 & 1.230 & 0.225 & $29 \%$ & $47 \%$ \\
\hline Lease \% total CRE (\%) & $39 \%$ & $37 \%$ & -0.397 & 0.693 & $-18 \%$ & $5 \%$ \\
\hline Lease total as \% years profit (\%) & $76 \%$ & $64 \%$ & -0.407 & 0.686 & $-28 \%$ & $12 \%$ \\
\hline Owned CRE per FTE (£) & 15881 & 17119 & 0.361 & 0.720 & $29 \%$ & $49 \%$ \\
\hline Lease total per FTE (£) & 20942 & 9144 & -0.928 & 0.365 & $10 \%$ & $36 \%$ \\
\hline Total CRE per FTE (£) & 36823 & 26263 & -0.969 & 0.337 & $17 \%$ & $44 \%$ \\
\hline Retail & 19 companies & 4 companies & & & & \\
\hline Turnover (£’m) & 8336 & 3049 & -0.697 & 0.493 & $37 \%$ & $27 \%$ \\
\hline PBIT (£’m) & 368 & 239 & -0.399 & 0.694 & $2 \%$ & $-19 \%$ \\
\hline Profit margin (\%) & $7 \%$ & $12 \%$ & 1.662 & 0.111 & $2 \%$ & $-12 \%$ \\
\hline Net assets (£’m) & 2155 & 873 & -0.684 & 0.502 & $32 \%$ & $78 \%$ \\
\hline FTE's & 49720 & 24120 & -0.550 & 0.588 & $13 \%$ & $15 \%$ \\
\hline SPP (£’m) & 48 & 0 & N/A & N/A & $138 \%$ & N/A \\
\hline Freehold (£’m) & 2146 & 661 & -0.565 & 0.578 & $41 \%$ & $8 \%$ \\
\hline Lease total (£’m) & 2546 & 1304 & -0.605 & 0.552 & $24 \%$ & $42 \%$ \\
\hline Total CRE (£'m) & 4692 & 1965 & -0.600 & 0.555 & $31 \%$ & $28 \%$ \\
\hline Lease \% total CRE (\%) & $77 \%$ & $72 \%$ & -0.367 & 0.718 & $-1 \%$ & $1 \%$ \\
\hline Lease total as \% years profit (\%) & $-21 \%$ & $350 \%$ & 0.186 & 0.854 & $-102 \%$ & $13 \%$ \\
\hline Owned CRE per FTE $(\mathfrak{E})$ & 17922 & 13596 & -0.363 & 0.720 & $23 \%$ & $0 \%$ \\
\hline Lease total per FTE $(\mathfrak{E})$ & 68216 & 50094 & -0.598 & 0.556 & $-10 \%$ & $17 \%$ \\
\hline Total CRE per FTE (£) & 86138 & 63689 & -0.728 & 0.474 & $-5 \%$ & $13 \%$ \\
\hline
\end{tabular}


Table 2. Mean values and t-test of change for variables 2007 to $2014(n=170)$

\begin{tabular}{|c|c|c|c|c|}
\hline Change 2007 to 2014 & SPP & Non-SPP & $t$-value & p-value \\
\hline All companies & 99 companies & 71 companies & & \\
\hline Turnover & $65.9 \%$ & $98.1 \%$ & 2.345 & $0.02^{\star *}$ \\
\hline Profit & $180.3 \%$ & $132.1 \%$ & -0.350 & 0.727 \\
\hline Profit margin & $28.7 \%$ & $29.2 \%$ & 0.012 & 0.991 \\
\hline Net assets & $5.5 \%$ & $142.3 \%$ & 1.679 & $0.095^{*}$ \\
\hline FTE's & $41.7 \%$ & $55.4 \%$ & 0.948 & 0.344 \\
\hline Freehold & $143.2 \%$ & $394.6 \%$ & 1.099 & 0.274 \\
\hline Lease total & $83.4 \%$ & $692.0 \%$ & 1.635 & 0.107 \\
\hline Total CRE & $83.4 \%$ & $609.4 \%$ & 1.458 & 0.149 \\
\hline Leases as \% total CRE & $-2.2 \%$ & $64.3 \%$ & 2.005 & $0.049^{* *}$ \\
\hline Lease total as $\%$ years profit & $-77.1 \%$ & $-619.4 \%$ & -0.542 & 0.589 \\
\hline Owned CRE per FTE & $105.9 \%$ & $134.8 \%$ & 0.305 & 0.761 \\
\hline Lease total per FTE & $43.3 \%$ & $284.3 \%$ & 1.816 & $0.073^{*}$ \\
\hline Total CRE per FTE & $42.9 \%$ & $209.6 \%$ & 1.328 & 0.188 \\
\hline Finance & 24 companies & 15 companies & & \\
\hline Turnover & $75.0 \%$ & $104.6 \%$ & 0.997 & 0.325 \\
\hline Profit & $96.8 \%$ & $75.2 \%$ & -0.299 & 0.767 \\
\hline Profit margin & $-7.2 \%$ & $-12.3 \%$ & -0.254 & 0.801 \\
\hline Net assets & $109.0 \%$ & $100.8 \%$ & -0.139 & 0.890 \\
\hline FTE's & $77.8 \%$ & $89.4 \%$ & 0.228 & 0.821 \\
\hline Freehold & $13.8 \%$ & $249.4 \%$ & 1.573 & 0.165 \\
\hline Lease total & $215.5 \%$ & $850.6 \%$ & 1.551 & 0.140 \\
\hline Total CRE & $218.4 \%$ & $862.4 \%$ & 1.466 & 0.161 \\
\hline Leases as \% total CRE & $-4.1 \%$ & $117.4 \%$ & 0.964 & 0.352 \\
\hline Lease total as \% years profit & $128.4 \%$ & $948.5 \%$ & 1.525 & 0.151 \\
\hline Owned CRE per FTE & $7.3 \%$ & $113.7 \%$ & 1.292 & 0.236 \\
\hline Lease total per FTE & $143.3 \%$ & $300.3 \%$ & 0.957 & 0.345 \\
\hline Total CRE per FTE & $147.3 \%$ & $285.3 \%$ & 0.815 & 0.420 \\
\hline Professional services & 20 companies & 9 companies & & \\
\hline Turnover & $64.7 \%$ & $67.4 \%$ & 0.111 & 0.912 \\
\hline Profit & $29.4 \%$ & $63.8 \%$ & 0.348 & 0.731 \\
\hline Profit margin & $-25.9 \%$ & $62.2 \%$ & 1.067 & 0.295 \\
\hline Net assets & $-145.0 \%$ & $136.3 \%$ & 0.606 & 0.550 \\
\hline FTE's & $35,0 \%$ & $47.8 \%$ & 0.549 & 0.587 \\
\hline Freehold & $64.6 \%$ & $38.3 \%$ & -0.326 & 0.747 \\
\hline Lease total & $51.9 \%$ & $95.3 \%$ & 0.966 & 0.343 \\
\hline Total CRE & $50.2 \%$ & $60.2 \%$ & 0.283 & 0.779 \\
\hline Leases as \% total CRE & $-0.3 \%$ & $17.7 \%$ & 1.816 & $0.080^{*}$ \\
\hline Lease total as $\%$ years profit & $9.0 \%$ & $48.4 \%$ & 1.05 & 0.303 \\
\hline Owned CRE per FTE & $30.4 \%$ & $-10.7 \%$ & -1.081 & 0.290 \\
\hline Lease total per FTE & $32.9 \%$ & $28.9 \%$ & -0.104 & 0.918 \\
\hline Total CRE per FTE & $26.5 \%$ & $5.8 \%$ & -1.115 & 0.275 \\
\hline TMT & 17 companies & 8 companies & & \\
\hline Turnover & $45.7 \%$ & $225.2 \%$ & 3.588 & $0.002^{\star \star \star x}$ \\
\hline Profit & $720.2 \%$ & $240.2 \%$ & -0.498 & 0.623 \\
\hline Profit margin & $206.4 \%$ & $5.6 \%$ & -0.757 & 0.457 \\
\hline Net assets & $-18.5 \%$ & $300.8 \%$ & 3.539 & $0.002^{\star \star \star x}$ \\
\hline FTE's & $22.5 \%$ & $154.7 \%$ & 3.202 & $0.012^{\star *}$ \\
\hline Freehold & $266.9 \%$ & $2376.8 \%$ & 1.128 & 0.296 \\
\hline Lease total & $28.3 \%$ & $3374.6 \%$ & 1.062 & 0.324 \\
\hline Total CRE & $29.3 \%$ & $3360.0 \%$ & 1.083 & 0.314 \\
\hline
\end{tabular}


End of Table 2

\begin{tabular}{|c|c|c|c|c|}
\hline Change 2007 to 2014 & SPP & Non-SPP & $t$-value & $\mathrm{p}$-value \\
\hline Leases as \% total CRE & $0.3 \%$ & $21.7 \%$ & 0.879 & 0.404 \\
\hline Lease total as $\%$ years profit & $-361.7 \%$ & $-7614.0 \%$ & 0.404 & 0.436 \\
\hline Owned CRE per FTE & $327.1 \%$ & $719.6 \%$ & 0.658 & 0.519 \\
\hline Lease total per FTE & $-1.1 \%$ & $1136.2 \%$ & 1.046 & 0.330 \\
\hline Total CRE per FTE & $-0.7 \%$ & $1100.6 \%$ & 1.036 & 0.335 \\
\hline Manufacturing & 19 companies & 35 companies & & \\
\hline Turnover & $87.3 \%$ & $75.5 \%$ & -0.528 & 0.599 \\
\hline Profit & $95.0 \%$ & $156.1 \%$ & 0.650 & 0.518 \\
\hline Profit margin & $-4.0 \%$ & $48.3 \%$ & 1.088 & 0.282 \\
\hline Net assets & $-14.0 \%$ & $126.8 \%$ & 1.504 & 0.139 \\
\hline FTE's & $33.8 \%$ & $22.5 \%$ & -0.795 & 0.430 \\
\hline Freehold & $88.8 \%$ & $73.8 \%$ & -0.476 & 0.636 \\
\hline Lease total & $24.5 \%$ & $242.3 \%$ & 2.271 & $0.029^{* *}$ \\
\hline Total CRE & $48.0 \%$ & $77.7 \%$ & 1.160 & 0.251 \\
\hline Leases as \% total CRE & $-11.1 \%$ & $72.0 \%$ & 1.393 & 0.169 \\
\hline Lease total as \% years profit & $-13.7 \%$ & $109.0 \%$ & 2.082 & $0.044^{\star \star}$ \\
\hline Owned CRE per FTE & $43.8 \%$ & $47.2 \%$ & 0.205 & 0.839 \\
\hline Lease total per FTE & $1.6 \%$ & $180.5 \%$ & 2.499 & $0.017^{\star *}$ \\
\hline Total CRE per FTE & $12.4 \%$ & $49.1 \%$ & 3.072 & $0.003^{* * *}$ \\
\hline Retail & 19 companies & 4 companies & & \\
\hline Turnover & $52.3 \%$ & $85.3 \%$ & 0.888 & 0.384 \\
\hline Profit & $46.5 \%$ & $72.0 \%$ & 0.384 & 0.705 \\
\hline Profit margin & $5.0 \%$ & $-10.4 \%$ & -0.343 & 0.735 \\
\hline Net assets & $74.0 \%$ & $130.7 \%$ & 0.447 & 0.660 \\
\hline FTE's & $28.1 \%$ & $35.2 \%$ & 0.243 & 0.810 \\
\hline Freehold & $306.8 \%$ & $32.6 \%$ & -0.428 & 0.673 \\
\hline Lease total & $64.7 \%$ & $48.6 \%$ & -0.161 & 0.873 \\
\hline Total CRE & $39.0 \%$ & $47.3 \%$ & 0.148 & 0.884 \\
\hline Leases as \% total CRE & $4.9 \%$ & $0.8 \%$ & -0.327 & 0.747 \\
\hline Lease total as \% years profit & $-225.3 \%$ & $7.2 \%$ & 0.827 & 0.418 \\
\hline Owned CRE per FTE & $184.7 \%$ & $5.6 \%$ & -0.434 & 0.669 \\
\hline Lease total per FTE & $14.6 \%$ & $8.2 \%$ & -0.189 & 0.852 \\
\hline Total CRE per FTE & $3.5 \%$ & $7.0 \%$ & 0.187 & 0.854 \\
\hline
\end{tabular}

All Companies: No financial metric in 2014 indicated a significant difference between the two groups, although the net assets metric was close $(p=0.106)$ to significant, indicating that SPP companies might have a larger asset base than non-SPP ones. Significant differences in CRE metrics between SPP and non-SPP companies in 2014 were identified for the metrics of lease total; total CRE and leases as a percentage of total CRE. In all cases the mean is larger for the SPP than the non-SPP group. Thus, SPP companies have a larger CRE portfolio with a greater emphasis on leasing than non-SPP companies. Looking at change over the eight years the non-SPP companies grew their turnover and net assets together with increased leases as a percentage of total CRE and the cost of leases per FTE more than SPP ones. The lease total $(\mathrm{p}=0.107)$ supports the view that non-SPP companies are expanding their business and with it their leased CRE at a greater rate than SPP ones. The increase in the SPP itself rose from $£ 15 \mathrm{~m}$ to $£ 37 \mathrm{~m}$, a $151 \%$ increase.
Finance: Financial measures showed no difference between SPP and non-SPP companies. The lease total as a percentage of profit was significantly higher for SPP companies, indicating a longer commitment to leases relative to profit generation. No difference was found on the change measure for financial metrics or CRE measures. The SPP rose by $218 \%$ from 2007 to $£ 44 \mathrm{~m}$ in 2014, the largest rise of all categories, but was not the largest absolute sum.

Professional Services: The analysis did not generate any significant differences between the SPP and non-SPP means for 2014. The change in leases as a percentage of total CRE was significant indicating that non-SPP companies have increased the proportion of total CRE they lease more than SPP companies. The amount of surplus leasehold space grew by $41 \%$ to $£ 14 \mathrm{~m}$.

TMT: Turnover and FTE's are larger for SPP companies and, no doubt to facilitate those additional people, the total CRE is larger, primarily through leasing (lease total 
$\mathrm{p}=0.100)$. Turnover, net assets, and FTE's all increased more for non-SPP companies over the period, although that is not reflected in the CRE change metrics. The category grew its SPP by $167 \%$ to $£ 73 \mathrm{~m}$, the highest absolute SPP sum across all categories.

Manufacturing: This is the only category where the number of non-SPP companies (35) exceeds the SPP ones (19). There are no differences between SPP and non-SPP companies for financial or CRE measures in 2014. Over the eight years the change for non-SPP companies of CRE metrics of lease total; lease total as a percentage of profits; lease total per FTE and total CRE per FTE increased more than SPP companies. This indicates that non-SPP companies are increasing their leases at a greater rate in absolute terms and relative terms, but the change is not matched by a significant change in business metrics. That would suggest that expansion in leases is speculative, rather than reacting to actual growth. The SPP total grew by $121 \%$ to $£ 9 \mathrm{~m}$, the smallest absolute sum of all categories. The larger proportion of non-SPP companies in this category (65\% against $42 \%$ for All Companies) may reflect the high CRE capital investment (buildings, their adaptation and the production plant contained in them), which could mean that only a significant long-term decline would trigger lease closures.

Retail: This has the smallest non-SPP sector (4 companies) which as mentioned earlier is regarded as very small $(\mathrm{N}<5)$ (de Winter, 2013), and would raise the question of validity on the test outcomes, but the $t$-test did not identify any differences. SPP jumped in 2009, 2013 and 2014, reflecting a decline in profits and suggesting an inverse relationship with profit levels, the total in 2014 being $£ 48 \mathrm{~m}$ $(+138 \%)$.

Taking a broad view and recognising that the $t$-test does not identify all the differences as significant, the data and analysis indicates that there are differences in the profiles for those that make an SPP and those that do not (Q1). SPP companies are generally larger, based on turnover, net assets and FTE's, but generate lower profits from a larger CRE portfolio. Over the eight years non-SPP companies have grown their business and expanded their $\mathrm{CRE}$ at a greater rate, in particular their leased space. The profile of a smaller CRE portfolio could suggest that the non-SPP group are more agile and flexible with dynamic alignment capability and able to react more quickly to business change, thereby exploiting transient competitive advantage opportunities (McGrath, 2013) or blue oceans (Kim \& Maugborgne, 2015). This could reflect a smaller organisation, certainly in terms of FTE's, which may mean they have flatter management structures. If so, they are more likely to benefit from iterative learning loops (Mintzberg et al., 2009) and the key decision-makers may have greater visibility of CRE issues (Greenhalgh, 2008; Nunnington \& Haynes, 2011).

The CRE analysis appears to be counter to the generally accepted view that space consumption has declined with new ways of working as the increased cost commit- ment indicates space is not being reduced. There is an argument that the cost increase could reflect rent reviews, but the period 2007 to 2014 saw significant falls in rental values because of the impact of the recession. An alternative explanation might be that businesses have built expansion space into their portfolios. However, that is unlikely for a few reasons. During the period business profits were under pressure (Figure 1) and there was considerable uncertainty in the world. Taking space and immediately designating it as surplus would hit profits even harder and would be unpalatable to investors. Secondly it would be something that auditors would not approve of because it breaches IAS37 requirements. Table 1 indicates that the SPP companies have a significantly higher commitment for CRE for various measures, including the commitment per FTE and the lease commitment expressed as a proportion of profit.

One potential explanation of the increase in freehold ownership is that the recession resulted in a number of forced sellers of commercial property and occupiers could have exploited the depressed market by acquiring freeholds at relatively low prices.

\subsection{Auto-regression model results questions 2,3 and 4}

The regression model of the best fit business metrics (Q2) was run following the multicollinearity checks and the output is in Table 3 . Those business metrics that were excluded due to multicollinearity are indicated in the table. The previous year metrics for turnover, profit and FTE were excluded across all categories and therefore have not been included in the table.

The regression models for business metrics show a good fit of data for most of the categories, with an $\mathrm{R}^{2} \mathrm{Ad}-$ justed over 0.750 (bar Manufacturing - 0.458).

Following the multicollinearity exercise the predominant significant metric was turnover, which showed a relationship with SPP for All Companies, TMT and Retail. Manufacturing had a significant positive relationship between FTE and SPP. Profit exhibited a significant negative relationship with SPP for All Companies and TMT, whilst the negative relationship for Finance was very close to the significance threshold of $\mathrm{p}<0.1(0.104)$. Profit margins had a negative relationship for Retail and positive for TMT. Professional Services did not exhibit any significant relationships for SPP with business metrics. The regression models anticipated a negative relationship between the SPP and all the business metrics as per Q2, however, only the relationship between SPP and profit was as anticipated. Both turnover and FTE had positive relationships, the converse of what had been hypothesised, whilst profit margins provide contradictory results.

The hypothesised inverse relationship between profits and SPP is supported: as profits decline operational space is closed and the provision for surplus property is increased. The expectation that a decline in revenue and/ or profit margin and/or FTE will see the SPP increase is 


\begin{tabular}{|c|c|c|c|c|c|c|c|c|c|c|c|c|c|c|c|c|c|c|c|c|c|c|c|c|}
\hline $\begin{array}{l}\text { 育 } \\
\text { 总 } \\
\text { 品 }\end{array}$ & & $\begin{array}{l}0 \\
0 \\
0 \\
0\end{array}$ & $\begin{array}{l}\infty \\
0 \\
0 \\
0\end{array}$ & $\begin{array}{l}\infty \\
\tilde{\sigma} \\
\hat{o}\end{array}$ & & $\stackrel{m}{m}$ & $\begin{array}{l}\overrightarrow{0} \\
\hat{O}\end{array}$ & \begin{tabular}{l} 
H \\
\multirow{+}{*}{} \\
0
\end{tabular} & & $\begin{array}{l}\vec{m} \\
\overrightarrow{+} \\
+\end{array}$ & $\begin{array}{c}\overrightarrow{2} \\
\stackrel{i}{i}\end{array}$ & $\begin{array}{l}0 \\
\stackrel{0}{0} \\
0\end{array}$ & & 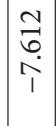 & $\begin{array}{l}n \\
\hat{L} \\
\hat{n} \\
0 \\
1\end{array}$ & $\begin{array}{l}\vec{\infty} \\
0 \\
0 \\
0\end{array}$ & & 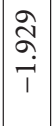 & $\begin{array}{l}n \\
\overrightarrow{0} \\
0 \\
i\end{array}$ & $\begin{array}{l}\text { ?운 } \\
\text { th? }\end{array}$ & & 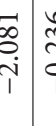 & $\begin{array}{l}0 \\
\vdots \\
\vdots \\
1\end{array}$ & \\
\hline 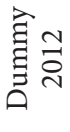 & & $\mid \begin{array}{l}0 \\
\hat{\infty} \\
0 \\
0 \\
0\end{array}$ & $\underset{\stackrel{L}{f}}{\stackrel{f}{-}}$ & $\underset{⿱}{\stackrel{q}{0}}$ & & $\begin{array}{l}\infty \\
\infty \\
\infty \\
i \\
i\end{array}$ & 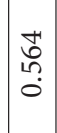 & 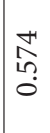 & & $\begin{array}{c}\infty \\
\infty \\
\infty \\
\dot{p} \\
\dot{p}\end{array}$ & 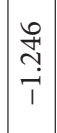 & $\stackrel{n}{N}$ & & $\begin{array}{l}\hat{\gamma} \\
m \\
m \\
-\end{array}$ & $\begin{array}{l}L \\
\infty \\
\infty \\
0 \\
0\end{array}$ & $\begin{array}{l}\hat{1} \\
\hat{\sigma} \\
0\end{array}$ & & 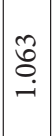 & $\begin{array}{c}9 \\
\text { fr } \\
0 \\
0\end{array}$ & 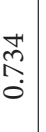 & & 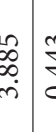 & 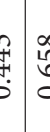 & \\
\hline $\begin{array}{l}\text { 裏च } \\
\text { 言 }\end{array}$ & & $\begin{array}{l}0 \\
\infty \\
\infty \\
i \\
i \\
T\end{array}$ & $\mid \begin{array}{c}0 \\
0 \\
10 \\
0 \\
1\end{array}$ & $\begin{array}{l}10 \\
\vdots \\
0 \\
0\end{array}$ & & 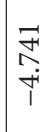 & \begin{tabular}{l}
$\infty$ \\
1 \\
\multirow{1}{0}{} \\
0 \\
1 \\
1
\end{tabular} & \begin{tabular}{l}
$\infty$ \\
\multirow{0}{0}{} \\
0 \\
0
\end{tabular} & & $\begin{array}{l}\vec{\partial} \\
\dot{p}\end{array}$ & $\begin{array}{l}\vec{\sigma} \\
\hat{i} \\
i\end{array}$ & $\stackrel{\pi}{\pi}$ & & 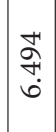 & †ิ & $\mid \begin{array}{l}0 \\
\hat{\sigma} \\
0\end{array}$ & & $\begin{array}{l}\widetilde{J} \\
\stackrel{L}{i} \\
i\end{array}$ & $\begin{array}{c}\stackrel{\sim}{1} \\
\infty \\
0 \\
0\end{array}$ & $\bar{F}$ & & 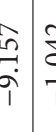 & $\begin{array}{l}y^{\prime} \\
\dot{1} \\
\end{array}$ & \\
\hline 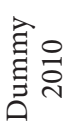 & & $\mid \begin{array}{l}0 \\
\infty \\
+ \\
i \\
i\end{array}$ & $\mid \begin{array}{c}\vec{J} \\
\tilde{C} \\
0\end{array}$ & $\begin{array}{l}8 \\
0 \\
0 \\
0\end{array}$ & & 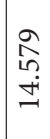 & 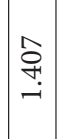 & $\begin{array}{l}\overrightarrow{0} \\
\stackrel{0}{0}\end{array}$ & & $\begin{array}{l}\underset{F}{*} \\
\underset{-}{*}\end{array}$ & $\begin{array}{l}l \\
0 \\
0 \\
0 \\
0\end{array}$ & $\begin{array}{l}\tilde{2} \\
\hat{h} \\
0\end{array}$ & & $\begin{array}{l}m \\
\infty \\
\stackrel{1}{a} \\
\hat{i}\end{array}$ & $\mid \begin{array}{l}0 \\
\infty \\
0 \\
0 \\
i\end{array}$ & $\begin{array}{l}\text { Jै } \\
\text { के } \\
0\end{array}$ & & $\frac{\hat{a}}{\stackrel{0}{0}}$ & 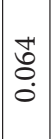 & $\begin{array}{l}\text { \&े } \\
\text { ô }\end{array}$ & $\begin{array}{l}\stackrel{2}{2} \\
\stackrel{2}{2} \\
1\end{array}$ & 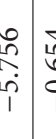 & 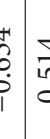 & \\
\hline 言 & & $\stackrel{\Xi}{\exists}$ & $\mid \begin{array}{c}\tilde{n} \\
\tilde{N} \\
0 \\
i\end{array}$ & $\begin{array}{c}\vec{t} \\
\infty \\
0 \\
0\end{array}$ & & $\frac{\vec{\sigma}}{\dot{m}}$ & \begin{tabular}{|l}
$\infty$ \\
0 \\
0 \\
0 \\
0
\end{tabular} & 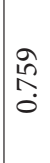 & & $\begin{array}{l}\stackrel{\vartheta}{\vec{i}} \\
\vec{i}\end{array}$ & $\begin{array}{l}0 \\
2 \\
0 \\
i \\
1\end{array}$ & $\begin{array}{l}\vec{\infty} \\
\stackrel{0}{0} \\
0\end{array}$ & & 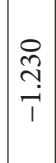 & $\begin{array}{l}\vec{a} \\
\dot{0} \\
i\end{array}$ & ふ̊. & & 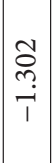 & $\begin{array}{l}\vec{\Im} \\
\stackrel{\Im}{0} \\
1\end{array}$ & $\begin{array}{l}n \\
\hat{b} \\
0 \\
0\end{array}$ & & \begin{tabular}{c|c}
\multirow{2}{c}{} & \multicolumn{2}{c}{} \\
$\infty$ \\
1 \\
1
\end{tabular} & 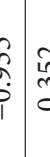 & \\
\hline $\begin{array}{l}\text { 言 } \\
\text { 豆 } \\
\text { ¿ }\end{array}$ & & $\begin{array}{l}\stackrel{0}{1} \\
\stackrel{i}{i}\end{array}$ & $\begin{array}{c}\stackrel{f}{f} \\
0\end{array}$ & 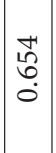 & & సิ & $\begin{array}{l}0 \\
0 \\
0 \\
0\end{array}$ & స్ & & 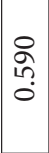 & $\begin{array}{l}2 \\
\stackrel{\sigma}{0}\end{array}$ & $\begin{array}{l}\infty \\
\infty \\
\infty \\
\infty \\
0\end{array}$ & & 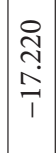 & 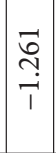 & 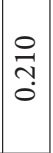 & & 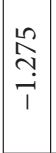 & 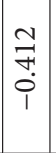 & 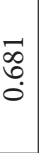 & $\begin{array}{l}\overrightarrow{0} \\
\dot{0} \\
\dot{m}\end{array}$ & 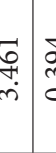 & 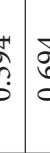 & \\
\hline 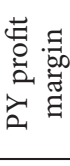 & & 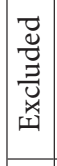 & & & & 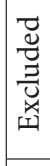 & & & & 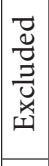 & & & & $\mid \begin{array}{l}\infty \\
\infty \\
0 \\
\dot{m} \\
\dot{p}\end{array}$ & 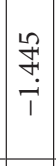 & $\vec{n}$ & & 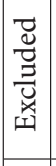 & & & $\begin{array}{l}\vec{z} \\
\vec{y} \\
\vec{z} \\
\tilde{u} \\
\underline{y}\end{array}$ & 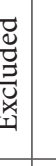 & & \\
\hline 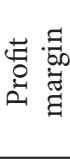 & & $\mid \begin{array}{c}\tilde{\omega} \\
\tilde{\omega} \\
0\end{array}$ & \begin{tabular}{l}
$\infty$ \\
$\stackrel{0}{0}$ \\
\hdashline
\end{tabular} & $\begin{array}{l}0 \\
\infty \\
0 \\
0\end{array}$ & & 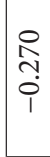 & $\begin{array}{l}+ \\
0 \\
0 \\
0 \\
i\end{array}$ & $\begin{array}{l}\stackrel{2}{\alpha} \\
\text { o. }\end{array}$ & & 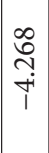 & $\begin{array}{l}0 \\
0 \\
0 \\
0 \\
0\end{array}$ & $\begin{array}{l}\infty \\
\infty \\
0 \\
0 \\
0\end{array}$ & & 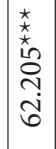 & $\stackrel{\vec{\Omega}}{\stackrel{i}{i}}$ & o & & $\begin{array}{l}\vec{F} \\
\stackrel{2}{n} \\
\vec{T} \\
T\end{array}$ & $\begin{array}{c}\infty \\
\infty \\
-1 \\
-1\end{array}$ & तु. & 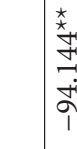 & 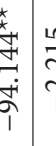 & 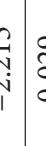 & \\
\hline 毁 & & 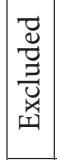 & & & & 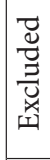 & & & & 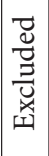 & & & & 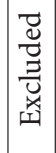 & & & & 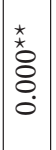 & ஓ & $\begin{array}{l}\text { के } \\
\text { ¿े }\end{array}$ & & & & \\
\hline $\begin{array}{l}\overrightarrow{\vec{D}} \\
\text { D } \\
\text {. }\end{array}$ & & 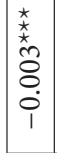 & $\begin{array}{l}\stackrel{2}{2} \\
\stackrel{9}{7} \\
1\end{array}$ & : & & $\begin{array}{l}\overrightarrow{8} \\
\dot{0} \\
i\end{array}$ & $\begin{array}{l}\hat{\hat{b}} \\
\stackrel{i}{i}\end{array}$ & 泀 & & 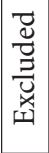 & & & & 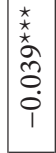 & 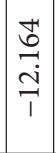 & \&̊ & & 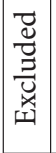 & & & 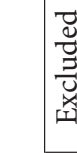 & 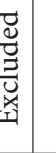 & & \\
\hline 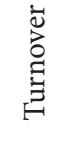 & $\begin{array}{l}\widehat{a} \\
\overrightarrow{0} \\
\dot{0} \\
v\end{array}$ & 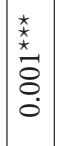 & 辛 & ڤ̊ & & 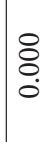 & 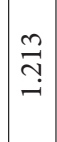 & $\begin{array}{l}\widehat{\hat{~}} \\
\text {. }\end{array}$ & $\widehat{\vec{\sigma}}$ & $\stackrel{8}{0}$ & $\begin{array}{l}\infty \\
\tilde{C} \\
0 \\
0\end{array}$ & $\begin{array}{l}\vec{n} \\
\hat{n} \\
0\end{array}$ & & 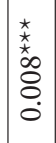 & $\begin{array}{l}\overrightarrow{\tilde{n}} \\
\ddot{\sim} \\
\vec{\sim}\end{array}$ & ஓ̊. & 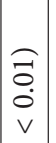 & \begin{tabular}{l}
8 \\
\hdashline \\
0 \\
0
\end{tabular} & $\mid \begin{array}{l}0 \\
\hat{o} \\
0 \\
i\end{array}$ & $\begin{array}{l}\stackrel{2}{\circ} \\
\text { के }\end{array}$ & 永 & 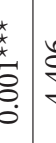 & 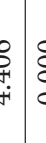 & \\
\hline 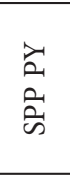 & 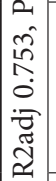 & 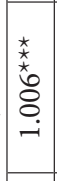 & $\begin{array}{l}+ \\
\infty \\
+ \\
\infty \\
\infty \\
\infty\end{array}$ & $\stackrel{8}{0}$ & $\begin{array}{l}\stackrel{0}{0} \\
v \\
n \\
\Delta \\
\Delta \\
0\end{array}$ & 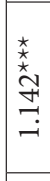 & $\begin{array}{l}\overrightarrow{2} \\
\tilde{n} \\
\grave{n}\end{array}$ & \& & 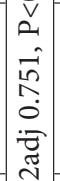 & 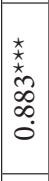 & 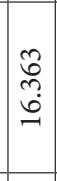 & ¿े & $\begin{array}{c}\widehat{\hat{\sigma}} \\
\dot{0} \\
v \\
0 \\
\hat{0} \\
\hat{0}\end{array}$ & 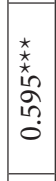 & $\begin{array}{c}m \\
\infty \\
0 \\
0 \\
- \\
-1\end{array}$ & ¿̊ & 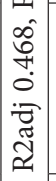 & 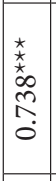 & 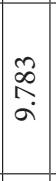 & $\begin{array}{l}\stackrel{8}{\circ} \\
\stackrel{\circ}{\circ}\end{array}$ & 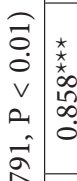 & 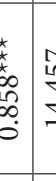 & 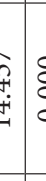 & \\
\hline $\begin{array}{l}\vec{E} \\
\vec{E} \\
\overrightarrow{0} \\
\tilde{0}\end{array}$ & 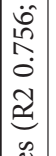 & $\frac{\Re}{\stackrel{\leftrightarrow}{i}}$ & $\mid \begin{array}{l}0 \\
0 \\
1 \\
0 \\
1 \\
1\end{array}$ & $\begin{array}{l}\hat{L} \\
\stackrel{L}{0} \\
0\end{array}$ & 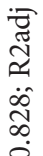 & $\begin{array}{l}\tilde{n} \\
\tilde{n} \\
\hat{1}\end{array}$ & $\begin{array}{l}\overrightarrow{1} \\
\hat{6} \\
0 \\
0 \\
1\end{array}$ & 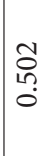 & 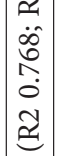 & 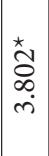 & 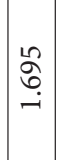 & ô & 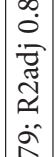 & \begin{tabular}{l}
$n$ \\
\multirow{+}{+}{} \\
$\dot{p}$ \\
1
\end{tabular} & $\begin{array}{c}\vec{F} \\
\text { D. } \\
i \\
1\end{array}$ & 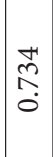 & 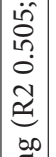 & 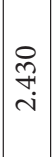 & $\overrightarrow{\widehat{\alpha}}$ & ڤે & 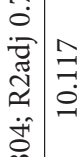 & 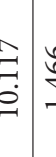 & 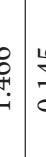 & \\
\hline & 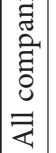 & 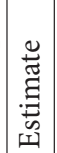 & 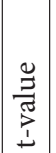 & 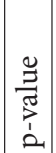 & 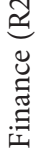 & 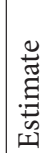 & 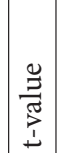 & 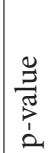 & 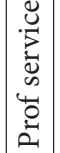 & 营 & 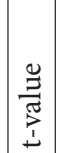 & | & 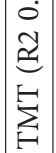 & 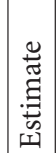 & 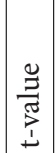 & 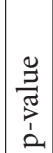 & 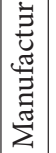 & 莺 & 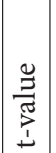 & 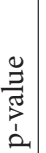 & 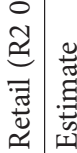 & 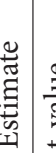 & 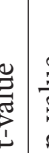 & \\
\hline
\end{tabular}


not supported. SPP is increasing when the business is expanding (as measured by turnover and FTE numbers), which indicates that CRE dynamic alignment capability is lacking. Businesses are not able to change the CRE profile without making an SPP.

The coefficients of the auto-regression term, the previous year SPP, are positive as expected (Table 3 ). The coefficient indicates the rate of decline of the surplus portfolio (Q3). The calculation 1/(1-coefficient) gives an estimate of how many years it takes before SPP tends towards zero. Three possible outcomes are projected: short term decline ( $<5$ years), long-term reduction ( $>5$ years) or continued increase in the SPP. The short-term decline group includes TMT (2.5 years) and Manufacturing (3.8 years), the longterm group comprises Retail (7.0 years) and Professional Services (8.6 years). Those indicating a continued growth in SPP consists of Finance (increasing by $14.2 \%$ per annum) and All Companies (growing at $0.6 \%$ per annum, albeit virtually a static position). Only TMT has the relationship between longevity SPP and CRE as proposed under Q3.

The expectation that SPP will be short term ( $<5$ years $)$ because the CRE has dynamic alignment capability was only identified for TMT and Manufacturing. For Retail and Professional Services, the slow rate of decline ( $>7$ years) indicates a limited CRE dynamic alignment capability. The Finance sector with its continued SPP expansion suggests a commitment to long term leases and a continuation of operational property closure. CRE portfolios do not appear in practice as agile as some expect them to be (Joroff \& Becker, 2017).

The same regression analysis process for business metrics was adopted for the lease metrics (Q4) (Table 4), including multicollinearity checks. Those lease metrics excluded due to multicollinearity are indicated in the table.

The data fit ( $\mathrm{R}^{2}$ Adjusted) is reasonably good $(>0.700)$ except for Manufacturing (0.436). Significant positive relationships were identified between SPP and leases $<1$ year are All Companies and Retail, whilst for leases $2-5$ years it is Finance. TMT had a positive relationship between SPP and the previous year leases $<1$ year but a negative one with the previous year leases $>5$ years. A positive previous year is akin to a negative relationship in the current year, whilst a positive one indicates negative for the current year. Therefore, TMT indicates a negative relationship for leases $<1$ year and a positive one for leases $>5$ years. Professional Services and Manufacturing did not exhibit any significant relationships between SPP and lease metrics. It had been anticipated that the relationship between SPP and leases $<5$ years would be negative (Q4); however, both are positive as short-term leases increase SPP increases.

The analysis of lease variables shows no evidence of there being an adjustment to a more flexible portfolio utilising the core and periphery approach (Gibson \& Lizieri, 1999). The general increase in the first periphery band could reflect a move to increasing the amount of shortterm space to provide more flexibility. The expectation would be a reduction in leases $>5$ years, but the evidence of a negative relationship between SPP and leases $>5$ years does not exist, rather for TMT it is positive relationship. The data (Table 1) and results (Table 4) suggest that business is expanding its CRE, especially of leases $<5$ years.

In summary, the broad pattern between SPP and both financial and CRE parameters is that SPP increases as profits decline (All Companies, Finance and TMT) and when turnover and/or FTE increases (All Companies, TMT, Manufacturing and Retail). This is reflected in the mean data values showing turnover at $+31 \%$, FTE's at $+7 \%$ and profits $-5 \%$. This would indicate a more complex set of relationships than envisaged.

The increase of SPP when profit declines, matches the original expectation reflecting closure of CRE when the business is under pressure. This decline in profits is not caused by the creation of the SPP itself, as explained in the methodology, an adjusted profit was used to remove the effect of any SPP increase. The increase of SPP when turnover and/or FTE's increase suggests a different rationale for closing space. If business is expanding production and/or increasing employment levels it may seek supplemental space and/or better space to deal with the capacity. Space that is replaced and added to the SPP indicates that portfolio's lack dynamic alignment capability. Supplemental space may be indicated by the increase in short-term leases for some business. What the analysis has shown is a lack of CRE dynamic alignment, which raises the question as to whether CRE agility has improved significantly over the last two decades (Joroff \& Becker, 2017).

The study identified that only in TMT did SPP revert to zero within three years. It also identified that $42 \%$ of the total survey sample (71 of 170 companies) did not make an SPP. Assuming the 71 companies do not have any surplus space, just $10 \%$ of the total sample indicate a possible dynamic alignment capability to facilitate CRE agility.

Business does not appear to be re-cycling surplus space. The expectation was that when additional space was needed the business would reduce surplus property first before taking new units. That does not appear to be the case, the commitment to CRE is increasing at a greater rate than the SPP is being removed. The additional CRE costs and the continuation of SPP will put pressure on future profit margins. As such it does raise the question as to how business is measuring the performance of its CRE and whether it is looking for added value from it.

\section{Limitations and recommendations}

A key benefit of using the data from the financial accounts of companies is the requirement that they report information in a set format, which provides consistency of data across companies. However, because the data provided in the company accounts is in this set format, it was not feasible for this research to provide supplementary data. For example, additional data on the number of units and total square metres would have been very beneficial. It would 


\begin{tabular}{|c|c|c|c|c|c|c|c|c|c|c|c|c|c|c|c|c|c|c|c|c|c|c|c|c|c|}
\hline 衰学 & & $\mid \begin{array}{l}8 \\
0 \\
10 \\
0 \\
1\end{array}$ & $\begin{array}{l}0 \\
0 \\
0 \\
1\end{array}$ & $\frac{\sigma}{\sigma}$ & & $\begin{array}{l}\overrightarrow{0} \\
\overrightarrow{0}\end{array}$ & $\begin{array}{l}8 \\
0 \\
0 \\
0\end{array}$ & $\begin{array}{l}0 \\
\text { in } \\
0 \\
0\end{array}$ & & 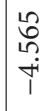 & $\begin{array}{l}\infty \\
\stackrel{\infty}{\infty}+ \\
\stackrel{+}{+}\end{array}$ & के & & $\begin{array}{l}\stackrel{n}{N} \\
\stackrel{n}{n}\end{array}$ & $\begin{array}{l}n \\
0 \\
0 \\
0\end{array}$ & $\mid \begin{array}{l}0 \\
0 \\
0 \\
0\end{array}$ & & $\begin{array}{l}\overrightarrow{\tilde{T}} \\
\stackrel{i}{1}\end{array}$ & 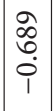 & ๙̃ & & $\begin{array}{l}\vec{\sigma} \\
\dot{+} \\
\dot{+}\end{array}$ & $\begin{array}{c}0 \\
\text { hn } \\
0 \\
0 \\
1\end{array}$ & $\begin{array}{l}n \\
\infty \\
0 \\
0 \\
0\end{array}$ & \\
\hline 昱 & & $\left|\begin{array}{l}n \\
\vec{\sim} \\
\tilde{b}\end{array}\right|$ & $\vec{m}$ & $\begin{array}{l}\infty \\
\stackrel{\infty}{\infty} \\
0 \\
0\end{array}$ & & $\begin{array}{l}\stackrel{N}{N} \\
\hat{n} \\
i n\end{array}$ & 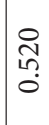 & $\begin{array}{l}\text { U' } \\
0 \\
0 \\
0\end{array}$ & & 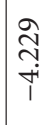 & $\stackrel{\substack{n \\
m \\
\rightarrow}}{\rightarrow}$ & 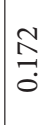 & & $\stackrel{\substack{m \\
-}}{\stackrel{m}{m}}$ & 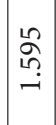 & $\underset{:}{\exists}$ & & $\mid \begin{array}{c}0 \\
\infty \\
0 \\
0\end{array}$ & 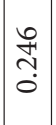 & 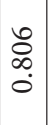 & & $\begin{array}{l}\text { ㅇ } \\
\text { a } \\
\text { a }\end{array}$ & $\begin{array}{l}\stackrel{2}{0} \\
\stackrel{0}{0} \\
\vdots\end{array}$ & $\begin{array}{l}\vec{b} \\
\grave{\sigma}\end{array}$ & \\
\hline 衰 & & $\mid \begin{array}{l}\hat{h} \\
\stackrel{+}{1} \\
i \\
1\end{array}$ & $\begin{array}{c}m \\
\hat{N} \\
\hat{i} \\
1\end{array}$ & $\begin{array}{l}\text { th } \\
\text { ț } \\
\stackrel{0}{0}\end{array}$ & & $\begin{array}{l}m \\
\hat{n} \\
\omega \\
1 \\
1\end{array}$ & $\begin{array}{l}\vec{n} \\
\text { ñ } \\
0 \\
1\end{array}$ & $\mid \begin{array}{l}0 \\
0 \\
0 \\
0 \\
0\end{array}$ & & $\begin{array}{l}\overrightarrow{0} \\
\stackrel{0}{+} \\
\stackrel{1}{1}\end{array}$ & 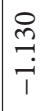 & $\begin{array}{l}\overrightarrow{0} \\
\stackrel{2}{0}\end{array}$ & & $\begin{array}{l}\stackrel{2}{2} \\
\infty \\
0\end{array}$ & $\begin{array}{l}0 \\
0 \\
0 \\
0\end{array}$ & $\begin{array}{l}\text { مू } \\
\text { o. }\end{array}$ & & $\begin{array}{l}\hat{\vec{N}} \\
\overrightarrow{\mathrm{N}}\end{array}$ & $\mid \begin{array}{l}2 \\
2 \\
\hat{2} \\
0\end{array}$ & 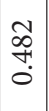 & & 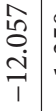 & 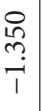 & $\begin{array}{l}\stackrel{2}{\hat{0}} \\
0\end{array}$ & \\
\hline 在。유 & & 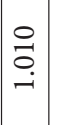 & $\begin{array}{l}\overrightarrow{1} \\
\stackrel{\sim}{0}\end{array}$ & $\begin{array}{c}0 \\
\infty \\
\infty \\
0 \\
0\end{array}$ & & 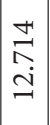 & 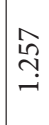 & $\overrightarrow{\tilde{N}}$ & & 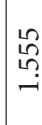 & $\begin{array}{l}\infty \\
0 \\
10 \\
0\end{array}$ & $\stackrel{m}{\sigma}$ & & 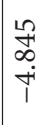 & $\begin{array}{l}\infty \\
\stackrel{\infty}{+} \\
\stackrel{i}{i}\end{array}$ & $\mid \begin{array}{l}\qquad \\
0 \\
\infty \\
0 \\
0 \\
0\end{array}$ & & \begin{tabular}{l}
$\infty$ \\
$\infty$ \\
\hdashline \\
0 \\
1
\end{tabular} & 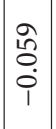 & $\begin{array}{l}\hat{\alpha} \\
\text { }\end{array}$ & & $\begin{array}{l}0 \\
0 \\
0 \\
0 \\
1\end{array}$ & 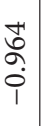 & $\widehat{\tilde{n}}$ & \\
\hline 言 & & 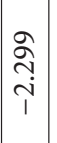 & \begin{tabular}{l}
$\infty$ \\
0 \\
\multirow{1}{0}{} \\
$i$ \\
1
\end{tabular} & 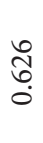 & & 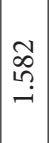 & $\begin{array}{l}10 \\
0 \\
0 \\
0\end{array}$ & $\begin{array}{l}0 \\
0 \\
0 \\
0 \\
0\end{array}$ & & 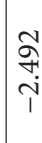 & $\begin{array}{l}m \\
\infty \\
\infty \\
0 \\
i\end{array}$ & 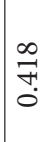 & & تُ & \begin{tabular}{|l|}
+ \\
0 \\
0 \\
0 \\
0
\end{tabular} & ભু & & $\begin{array}{l}\underset{N}{N} \\
\stackrel{i}{T}\end{array}$ & $\mid \begin{array}{l}0 \\
4 \\
n \\
0 \\
1\end{array}$ & $\begin{array}{l}0 \\
\infty \\
\infty \\
i \\
0\end{array}$ & & 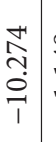 & $\begin{array}{c}\infty \\
\stackrel{+}{+} \\
\stackrel{i}{T} \\
\end{array}$ & 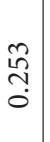 & \\
\hline $\begin{array}{l}\text { 量 } \\
\text { 号 }\end{array}$ & & $\left(\begin{array}{c}\mathcal{D} \\
\stackrel{1}{-} \\
-\end{array}\right.$ & $\begin{array}{l}\infty \\
0 \\
0 \\
0 \\
0\end{array}$ & $\begin{array}{l}\stackrel{2}{\circ} \\
\stackrel{1}{0}\end{array}$ & & 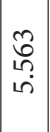 & $\begin{array}{l}0 \\
\text { hn } \\
0\end{array}$ & $\begin{array}{l}n \\
\infty \\
0 \\
0 \\
0\end{array}$ & & $\stackrel{m}{\tilde{m}}$ & $\begin{array}{l}2 \\
\stackrel{1}{0} \\
0\end{array}$ & $\frac{m}{\sigma}$ & & 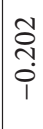 & $\begin{array}{l}0 \\
0 \\
0 \\
0 \\
i\end{array}$ & ठे & & 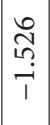 & 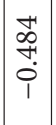 & $\begin{array}{l}\text { స్రి } \\
\text { : }\end{array}$ & & 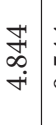 & 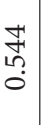 & $\begin{array}{l}\hat{\infty} \\
\text { ho } \\
0\end{array}$ & \\
\hline 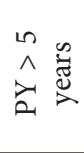 & & 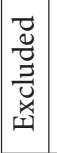 & & & & 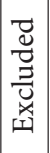 & & & & 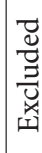 & & & & 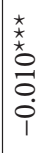 & \begin{tabular}{l}
$\stackrel{8}{2}$ \\
$\stackrel{1}{i}$ \\
\hdashline
\end{tabular} & ô & & 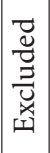 & & & & 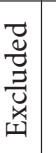 & & & \\
\hline 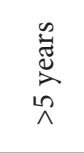 & & 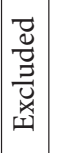 & & & & 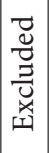 & & & & 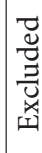 & & & & 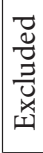 & & & & 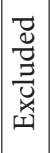 & & & & 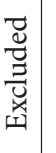 & & & \\
\hline 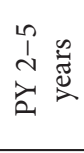 & & 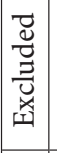 & & & & 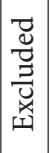 & & & & 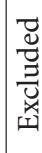 & & & & 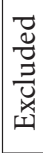 & & & & 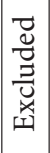 & & & & 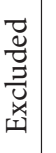 & & & \\
\hline 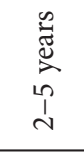 & & 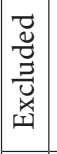 & & & & $\begin{array}{l}x \\
⿱ x \\
\vdots \\
\vdots \\
0 \\
0\end{array}$ & مُ & $\begin{array}{l}\infty \\
0 \\
0 \\
0\end{array}$ & & $\begin{array}{l}\overrightarrow{8} \\
\dot{0} \\
i\end{array}$ & $\begin{array}{l}0 \\
\infty \\
0 \\
0 \\
1\end{array}$ & $\begin{array}{l}2 \\
2 \\
0 \\
0 \\
0\end{array}$ & & 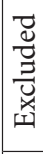 & & & & $\begin{array}{l}1 \\
\delta \\
0 \\
0\end{array}$ & $\begin{array}{l}2 \\
\hat{\sigma} \\
0 \\
0\end{array}$ & $\vec{\sigma}$ & & 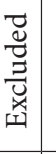 & & & \\
\hline $\begin{array}{l}\vec{v} \\
\vec{\Xi} \\
\vec{\varpi}\end{array}$ & & 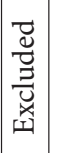 & & & & \begin{tabular}{|l}
$\vec{z}$ \\
$\vec{z}$ \\
$\vec{z}$ \\
$\bar{x}$ \\
$x$
\end{tabular} & & & & 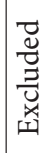 & & & & 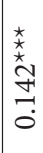 & 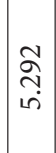 & 号 & & $\mid \begin{array}{l}\vec{y} \\
\vec{z} \\
\vec{z} \\
\bar{x} \\
x\end{array}$ & & & & 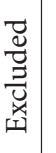 & & & \\
\hline $\begin{array}{l}\stackrel{\vec{\Xi}}{\Xi} \\
\vec{v} \\
\vec{v}\end{array}$ & $\begin{array}{l}\widehat{\partial} \\
\dot{0} \\
v \\
\Delta \\
\Delta\end{array}$ & 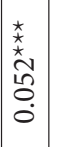 & $\begin{array}{l}\infty \\
\infty \\
\stackrel{1}{1} \\
\end{array}$ & $\stackrel{\square}{\circ}$ & $\widehat{\widehat{a}}$ & 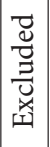 & & & $\begin{array}{l}\widehat{a} \\
\dot{0} \\
v \\
0\end{array}$ & 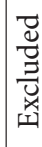 & & & & 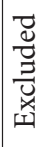 & & & $\begin{array}{l}a \\
0 \\
0 \\
v \\
0 \\
0\end{array}$ & 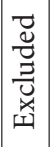 & & & $=$ & 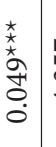 & $\begin{array}{c}\hat{n} \\
\stackrel{n}{\infty} \\
+ \\
+\end{array}$ & $\begin{array}{l}8 \\
\dot{0} \\
\circ\end{array}$ & \begin{tabular}{|l|}
$\vec{b}$ \\
$\dot{0}$ \\
$v$ \\
2 \\
$\Delta$
\end{tabular} \\
\hline $\begin{array}{l}\grave{\Delta} \\
\hat{a} \\
\ddot{\omega}\end{array}$ & 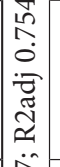 & 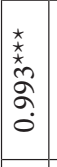 & $\begin{array}{l}m \\
\hat{\sigma} \\
\hat{n} \\
\hat{n}\end{array}$ & ڤ. & $\begin{array}{c}0 \\
v \\
0 \\
\infty \\
\infty \\
0 \\
0\end{array}$ & 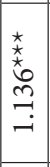 & $\stackrel{\substack{n\\
}}{\stackrel{2}{\sim}}$ & ¿̊. & 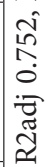 & 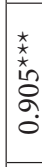 & \begin{tabular}{l}
$\infty$ \\
\multirow{+}{+}{} \\
$\stackrel{i}{+}$
\end{tabular} & $\stackrel{8}{\circ}$ & $\begin{array}{l}0 \\
\dot{0} \\
v \\
\text { v. } \\
\hat{0} \\
\hat{1} \\
\end{array}$ & 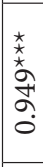 & 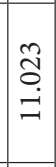 & $\stackrel{8}{\circ}$ & 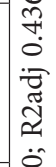 & 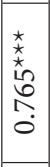 & $\mid \begin{array}{l}\hat{O} \\
0 \\
O \\
0\end{array}$ & $\stackrel{8}{\circ}$ & $\begin{array}{l}0 \\
0 \\
v \\
0 \\
0 \\
0 \\
1 \\
\end{array}$ & 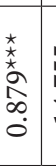 & 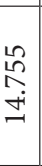 & & 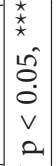 \\
\hline 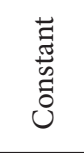 & 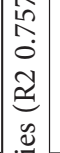 & $\begin{array}{l}\hat{\imath} \\
\hat{\sigma} \\
i \\
i\end{array}$ & $\begin{array}{l}\tilde{T} \\
0 \\
i \\
1\end{array}$ & $\begin{array}{l}\text { Fే } \\
\text { in } \\
0\end{array}$ & 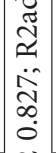 & $\begin{array}{c}\stackrel{N}{D} \\
\dot{10} \\
1\end{array}$ & $\begin{array}{l}\stackrel{2}{0} \\
0 \\
0 \\
1\end{array}$ & 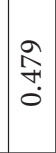 & $\begin{array}{l}\ddot{0} \\
\stackrel{0}{0} \\
\hat{0} \\
\tilde{z}\end{array}$ & $\begin{array}{l}\stackrel{\star}{さ} \\
\text { Oे } \\
\stackrel{+}{+}\end{array}$ & 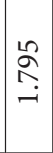 & $\begin{array}{l}\stackrel{n}{2} \\
0 \\
0\end{array}$ & 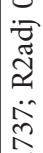 & $\begin{array}{l}n \\
\hat{h} \\
0 \\
i \\
1\end{array}$ & $\begin{array}{l}\text { 竍 } \\
\hat{n} \\
0 \\
i\end{array}$ & $\begin{array}{l}10 \\
\infty \\
1 \\
0 \\
0\end{array}$ & 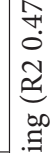 & $\begin{array}{c}\widetilde{\sigma} \\
\widetilde{\sigma} \\
\sim \\
\sim\end{array}$ & 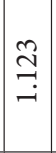 & 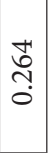 & 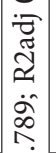 & $\stackrel{\stackrel{\leftrightarrow}{n}}{\stackrel{\sim}{i}}$ & 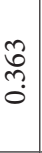 & & 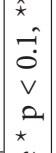 \\
\hline & 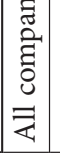 & 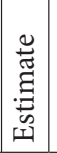 & 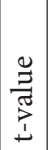 & 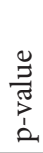 & 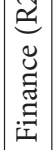 & 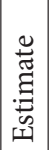 & 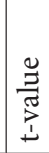 & 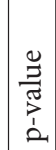 & 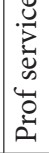 & 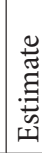 & 总 & 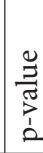 & $\begin{array}{l}\text { है } \\
\sum_{\text {I }}\end{array}$ & 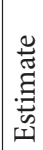 & 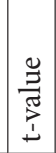 & 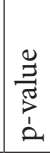 & 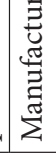 & 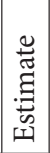 & 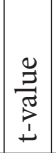 & 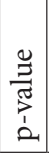 & 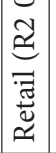 & 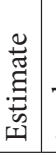 & 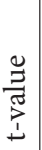 & 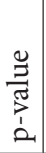 & 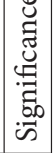 \\
\hline
\end{tabular}


enable a more extensive analysis of the CRE portfolio and link the spatial and financial elements. The lack of knowledge on the 71 non-SPP companies is a limitation of this study. The solution could be to approach those companies to seek to ascertain the status of surplus property throughout the eight years. However, getting cooperation and identifying personnel with appropriate knowledge across the period is likely to be difficult. The SPP forms part of the total provision and whilst the research could have considered the change in the total provision without directly approaching companies it would not be possible to draw any conclusions about the SPP from any increase in the total provision. The only option would be a study of the 71 companies individually. An alternative approach to compensate for the 71 companies could be an extension of the sample group. This could match the business sector profile of the 170 companies or it could resolve another limitation of the study by extending beyond quoted UK companies. The addition of unquoted UK companies might offset the disproportionate representation of financial services in the FTSE350, whilst including companies from other countries could provide a comparison of the impact of the UK lease term on dynamic alignment capability between countries.

It is possible that the relatively consistent growth in turnover over the eight-year period may have been a factor in the positive SPP relationship identified, rather than the projected inverse one. A more in-depth search on specific companies that have undergone volatility in turnover might prove enlightening, although the strength of the relationship between SPP and turnover would suggest any change in the results unlikely.

Short term occupational agreements that comprise the second periphery group are not identified in company accounts and therefore analysis of them has not been possible. It would be beneficial to research the extent to which business utilised pay as you go agreements and whether they were the first tranche of space closed when profits declined. Indeed, extending the research to look at the proportion of the total portfolio that pay as you go space comprises would extend the knowledge on CRE portfolio structuring and the capability for agility.

A feature of the UK economy over the last decade has been poor productivity (Guardian, 2016). Despite profits being depressed over the period $(-5 \%)$ there was a $7 \%$ increase in FTE numbers A further line of research is whether companies sought to avoid headcount reduction to ensure they had the right people for when the economic climate improved, but this does not explain the 19\% increase in CRE. An understanding of this dynamic might shed light on changes to the SPP and the relationship with CRE metrics, as this is another indication that is contrary to the notion that there is an ongoing reduction in space consumption. An additional consideration on the use of space use is not only the square metres of consumed but an examination of the CRE cost commitment (the total cost of CRE over the life of the lease) and how that relates to FTE's. This would provide both the absolute and relative costs of space. Research that considers the rationale of CRE change at a company level with case studies should provide additional knowledge on CRE decision-making and whether direct evidence can be found linking the size of company, the length of business strategy time horizon and the flexibility through the length of lease commitments.

What the research does illustrate is that surplus property is not a transitory effect of re-alignment. The analysis identifies that $90 \%$ of companies with an SPP still have the liability three years later, all things being equal. The realignment of CRE is not a seamless process as there appears to be obstacles in the way. Therefore, alignment models such as Lindholm et al. (2006) need to be adjusted to include the process of realignment itself and its consequential effects. Joroff and Becker (2017) suggested that CRE has become an agile asset over the last 25 years, indeed "agility has become an overarching objective" (Joroff \& Becker, 2017, p. 33). This research suggests that in practice CRE has not become agile. Whilst agility may be sought so far it does not appear to have been universally implemented. Consequently, there is a disconnect between what is being said about CRE agility and what the financial analysis shows. The suggestion that decision-making has become more holistic might apply in certain elements of the workplace, but not for the consequential effects of change for the portfolio.

There are hidden costs associated with surplus property management relating to risk, financial reporting compliance, etc. that impact on business performance. Decision-making techniques need to incorporate the consequences of creating surplus property to ensure they function adequately (Neely \& Bourne, 2000). The research indicates that decision making structures, such as the BSC (Kaplan \& Norton, 1996), and iterative learning processes (e.g. Mintzberg et al., 2009; Kay, 2010) need to include all CRE and surplus property aspects as part of the overall assessment.

What the research confirms is that CRE dynamic alignment capability has not been fully adopted by occupiers and the manner in which CRE change takes place and its inter-relationship with the SPP does not follow anticipated patterns. That would suggest that a line of research would be to investigate individual company decision-making processes. This would look to identify the factors considered by the decision-makers in dealing with CRE in times of change and adoption of a dynamic alignment capability.

This research provides a number of elements for practitioners to consider. Firstly, the need to review their CRE portfolio and ascertain its dynamic alignment capability. Allied to that is an understanding of the relative importance of individual units to the business allowing the CRE Manager to differentiate between core and periphery space. From that point there should be a CREAM strategy to build flexibility into the portfolio, for example, by means of break clauses or short-term leases to 
create CRE dynamic alignment capability that links with the status of each property. Together with an examination of CRE costs per FTE and to determine whether they are declining or are actually rising as new ways of working are implemented. The broader approach for the business involves developing management and decisionmaking systems that reflect the iterative, emergent nature of business strategy and acting to avoid the creation of a surplus property portfolio.

\section{Conclusions}

This paper has sought to understand how surplus leasehold property has changed and with it the inter-relationship with financial and CRE parameters over an eight-year period. It identified mixed evidence of dynamic alignment of CRE. CRE dynamic alignment capability appears to remain a theoretical concept for many organisations as this research indicates a lack of evidence of it in the financial reporting of CRE. Businesses appear to have used the SPP as a means of enabling them to react to business change because the portfolio lacks flexibility that short term leases and break clauses provide. This lack of dynamic alignment capability will hamper a company in responding to change arising from business or economic downturn or from opportunities. As such a lack of CRE agility will impair the ability of a business to exploit opportunities and respond to the effects of transient competitive advantage (McGrath, 2013) and/or blue ocean strategy (Kim \& Maugborgne, 2015) opportunities.

\section{References}

Apgar, M. (2009, November). What every leader should know about real estate. Harvard Business Review, 100-107.

Avis, M., \& Dent, P. (2004). The strategic management of surplus property in the NHS. Property Management, 22(4), 303-317. https://doi.org/10.1108/02637470410558161

Barney, J. B. (1991). Firm resources and sustained competitive advantage. Journal of Management, 17(1), 99-120. https://doi.org/10.1177/014920639101700108

Bingham, C. B., Eisenhardt, K. M., \& Furr, N. R. (2011). Which strategy when? MIT Sloan Management Review (Fall), 1-12.

Cooke, H. (2004). FRS12: guidance on implementation for corporate real estate managers. Journal of Corporate Real Estate, 6(4), 309-324. https://doi.org/10.1108/14630010410812450

Cooke, H., \& Appel-Meulenbroek, R. (2015). Impact of corporate real estate on UK business. In Proceedings of the 22nd ERES Conference, 24-27 June 2015. Istanbul, Turkey. https://doi.org/10.15396/eres2015_89

Cooke, H., \& Foster, A. L. (2016, August). Breaking up is hard to do. Estates Gazette, 27.

Cooke, H., \& Woodhead, S. (2008). Break strategy - the key to breaking out. Journal of Corporate Real Estate, 10(2), 110-120. https://doi.org/10.1108/14630010810905615

CoStar. (2012). Oxford agrees Cisco Green Park surrender. Retrieved from http://www.costar.co.uk/en/assets/news/2012/ July/Oxford-agrees-Cisco-Green-Park-surrender/

de Vries, J. C., de Jonge, H., \& van der Voordt, T. J. M. (2008). Impact of real estate interventions on organisational per- formance. Journal of Corporate Real Estate, 10(3), 208-223. https://doi.org/10.1108/14630010810922094

de Winter, J. C. F. (2013). Using the Students' $t$-test with extremely small sample sizes. Practical Assessment, Research \& Evaluation, 18(10), 1-12.

Gale, J., \& Case, F. (1989). A study of corporate real estate resource management. Journal of Real Estate Research, 4(3), 23-34

Gibler, K. M., \& Lindholm, A.-L. (2012). A test of corporate real estate strategies and operating decisions in support of core business strategies. Journal of Property Research, 29(10), 2548. https://doi.org/10.1080/09599916.2011.608470

Gibson, V. A., \& Lizieri, C. M. (1999). New business practices and the corporate property portfolio: how responsive is the UK property market? Journal of Property Research, 16(3), 201-218. https://doi.org/10.1080/095999199368111

Greenhalgh, P. (2008). An examination of business occupiers relocation decision making: distinguishing small \& large firm behaviour. Journal of Property Research, 25(2), 107-126. https://doi.org/10.1080/09599910802605368

Guardian. (2016). UK productivity gap widens to worst level since records began 18 February 2016. Retrieved from https://www. theguardian.com/business/2016/feb/18/uk-productivity-gapwidens-to-worst-level-since-records-began

Haynes, B., \& Nunnington, N. (2010). Corporate real estate asset management: strategy and implementation. Oxford: EG Books. https://doi.org/10.4324/9780080965222

Heywood, C. (2011). Approaches to aligning corporate real estate and organisational strategy. In Proceedings of the 18th ERES Conference, 15-18 June 2011. Eindhoven, The Netherlands.

Heywood, C., \& Arkesteijn, M. (2017). Alignment and theory in corporate real estate alignment models. International Journal of Strategic Property Management, 21(2), 144-158. https://doi.org/10.3846/1648715X.2016.1255274

Heywood, C., \& Arkesteijn, M. (2018). Analysing fourteen graphical representations of corporate real estate alignment models. Journal of Corporate Real Estate, 20(1), 16-40. https://doi.org/10.1108/JCRE-02-2017-0005

IAS37. (2001). International Accounting Standard 37: Provisions, contingent liabilities and contingent assets. London: International Accounting Standards Board.

Jensen, P. A., van der Voordt, T., \& Coenen, C. (Eds.) (2012). The added value of facilities management: concepts, findings and perspectives. Lyngby, Denmark: Centre for Facilities Management \& Polyteknisk Forlag.

Joroff, M., \& Becker, F. (2017, March). Exploiting change and uncertainty to drive corporate value. The Leader, 32-35.

Kämpf-Dern, A., \& Pfnür, A. (2014). Best practice, best model, best fit. Journal of Corporate Real Estate, 16(2), 97-125. https://doi.org/10.1108/JCRE-09-2013-0027

Kaplan, R. S., \& Norton, D. P. (1996). The balanced scorecard. Boston: Harvard Business School Press.

Kay, J. (2010). Obliquity. London: Profile Books.

Kim, W. C., \& Maugborgne, R. (2015). Blue ocean strategy (expanded ed.). Boston: Harvard Business School.

Krumm, P. J. M. M., \& de Vries, J. (2003). Value creation through the management of corporate real estate. Journal of Property Investment \& Finance, 21(1), 61-72. https://doi.org/10.1108/14635780310468310

Lindholm, A.-L., Gibler, K. M., \& Levainen, K. I. (2006). Modeling the value-adding attributes of real estate to the wealth maximization of the firm. Journal of Real Estate Research, 28(4), 445-475. 
Lizieri, C. M. (2003). Occupier requirements in commercial real estate markets. Urban Studies, 40(5-6), 1151-1169. https://doi.org/10.1080/0042098032000074353

MacIntosh, R., \& MacLean, D. (2015). Strategic management strategists at work. London: Palgrave.

Maiona, M. (2013). The revised lease accounting proposals: Aesop's fables revisited. Corporate Real Estate Journal, 3(1), 53-74.

McGrath, R. G. (2013). The end of competitive advantage. Boston: Harvard Business Review Press.

Mintzberg, H., Ahlstrand, B., \& Lampel, J. (2009). Strategy Safari (2nd ed.). Harlow: FT Prentice Hall.

Neely, A., \& Bourne, M. (2000). Why measurement initiatives fail. Measuring Business Excellence, 4(4), 3-7. https://doi.org/10.1108/13683040010362283

Nourse, H. O., \& Roulac, S. E. (1993). Linking real estate decisions to corporate strategy. Journal of Real Estate Research, 8(4), 475-494.

Nunnington, N., \& Haynes, B. P. (2011). Examining the building selection decision-making process within corporate relocations. Journal of Corporate Real Estate, 13(2), 109-121. https://doi.org/10.1108/14630011111136821
O'Mara, M. A. (1999). Strategy and place: managing corporate real estate and facilities for competitive advantage. New York: The Free Press.

Porter, M. E. (1985). Competitive advantage. New York: Free Press (extended edition in 1998 of the 1985 original).

Reeves, M., Levin, S., \& Ueda, D. (2016, January-February). The biology of corporate survival. Harvard Business Review, 4755.

Riratanaphong, C., \& Van der Voordt, T. (2015). Measuring the added value of workplace change: performance measurement in theory and practice. Facilities, 33(11/12), 773-792. https://doi.org/10.1108/F-12-2014-0095

Roulac, S. E. (2001). Corporate property strategy is integral to corporate business strategy. Journal of Real Estate Research, 22(1/2), 129-151.

Van der Voordt, T., Jensen, P. A., Hoendervanger, J. G., \& Bergsma, F. (2016). Value adding management (VAM) of buildings and facility services in four steps. Corporate Real Estate Journal, 6(1), 42-56.

Worthington, A. C., \& West, T. (2001). Economic value added: a review of the theoretical and empirical literature. Asian Review of Accounting, 19(9), 67-86. https://doi.org/10.1108/eb060736 